\title{
Research Paper \\ The Mediating Role of Sexual Self-Schema in the Relationship of Sexual Functioning With Sexual Satisfaction in Married Female College Students
}

\author{
Fatemeh Zargarinejad $^{1}$ D, Mehrnaz Ahmadi ${ }^{1}$ IID
}

1. Department of Psychology, Faculty of Education and Psychology, South Tehran Branch, Islamic Azad University, Tehran, Iran.

\begin{tabular}{l|l}
$\begin{array}{c}\text { Use vourdevice to scan } \\
\text { and read the article online }\end{array}$ \\
ing With Sexual Satisfaction in Married Female College Students .Iranian Journal of Psychiatry and Clinical Psychology. 2020; \\
25(4):412-427. http://dx.doi.org/10.32598/ijpcp.25.4.5 \\
doijhttp://dx.doi.org/10.32598/ijpcp.25.4.5
\end{tabular}

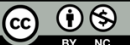

Received: 12 Dec 2018 Accepted: 31 Aug 2019 Available Online: 01 Jan 2020

Key words:

Sexual self-schema Sexual functioning, Sexual satisfaction, Married female students

\begin{abstract}
A B STRACT
Objectives This study aimed to examine the mediating role of sexual self-schema in the relationship of sexual functioning with sexual satisfaction in married female students.

Methods This is a descriptive correlational study. The study population consists of all married female students of Alzahra University and Islamic Azad University of South Tehran branch in 2017. Of these, 360 were selected by multistage cluster random sampling method. Data were collected using Women's Sexual Self-Schema Scale of Anderson and Cyranowski (1994), Female Sexual Function Index of Rosen et al. (2000), and Index of Sexual Satisfaction designed by Hudson (1993). Data were analyzed using descriptive statistics and using path analysis.

Results The Passionate-Romantic factor of sexual self-schema had a positive mediating role in relationship of sexual function with sexual satisfaction, while its Embarrassed-Conservative dimension had a negative mediating role $(\mathrm{P}<0.001)$. The factor of Open-Direct had no mediating role. Moreover, sexual functioning had a significant positive effect on Passionate-Romantic and Open-Direct dimension of sexual self-schema, while its effect on Embarrassed-Conservative was significantly negative. Furthermore, the Passionate-Romantic factor had a significant positive effect on sexual satisfaction, while the factor of Embarrassed-Conservative had a significant negative effect $(P<0.001)$. The factor of Open-Direct had no direct effect on sexual satisfaction. The effect of sexual functioning on sexual satisfaction was significantly negative $(\mathrm{P}<0.001)$.
\end{abstract}

Conclusion Sexual self-schema mediates the relationship of sexual functioning with sexual satisfaction.

\section{Extended Abstract}

\section{Introduction}

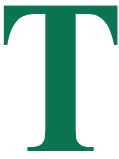

he study of sexual functioning and its physical and psychological components is one of the most important issues in studies related to family, relationships, and marriage [1]. Sexual functioning is one of the most important issues affecting marital satisfaction of couples. One of the most recent theories of sexual functioning has been introduced by Rosen et al. (2000), where six components have been defined for sexual functioning including sexual desire, sexual arousal, lubrication, orgasm, satisfaction and pain The factors than can affect the mental health of women in marital life, their sexual desire, sexual satisfaction, and sexual functioning are physiological, psychosocial, developmental and sexual responses [9]. Maladaptive thoughts and feedback play an important role in how information interpreted in sexual situations and ultimately, in experi-

* Corresponding Author:

Fatemeh Zargarinejad, MA.

Address: Department of Psychology, Faculty of Education and Psychology, South Tehran Branch, Islamic Azad University, Tehran, Iran.

Tel: +98 (912) 2217027

E-mail: $m$ ahmadi@azad.ac.ir 
ence, desire, arousal and orgasm. If the input stimulus is interpreted to cause negative thoughts, it may have different effects on one's sexual responses [11].

One of the key factors in assessing one's quality of life in general, and the quality and continuity of relationship marriage in particular, is sexual satisfaction in marital life [12]. Sexual satisfaction is a component associated with human sexuality that is considered as the last stage of the sexual response cycle [16]. Sexual satisfaction is an emotional response arising from the subjective evaluation of positive and negative dimensions related to sexual relationship [17]. Sexual satisfaction is not just a physical pleasure, but also includes satisfaction from sexual activity and emotional satisfaction [18]. Studies have shown that sexual Functioning has a significant relationship with sexual satisfaction [23, 34-36, 38]. Theoretical and empirical advances have led to a greater understanding of effective cognitive attitudes in marital relationships. One of the schemas involved in marital affairs is the sexual self- schema. Sexual self-schemas are cognitive generalizations about sexual aspects derived from one's past experiences manifested in current experiences, and influence the processing of social information related to sexual activity and guide sexual behavior. Selfconcept and positive sexual feedback can lead to satisfying sexual functioning, while negative sexual feedback along with conflicts and weakness can lead to distress, and sexual problems [42]. The present study aimed to investigate the mediating role of sexual self-schema in the relationship of sexual functioning with sexual satisfaction in married female college students.

\section{Methods}

This is a descriptive correlational study. The study population consisted of all married female students of Alzahra University and Islamic Azad University of South Tehran Branch. of these, 360 students were selected by multistage cluster random sampling method. Data were collected using Women's Sexual Self-Schema Scale of Anderson and Cyranowski (1994) [41], Female Sexual Function Index of Rosen et al. (2000) [4], and Index of Sexual Satisfaction designed by Hudson (1981) [56].

\section{Results}

To examine the mediating role of sexual self-schema in the relationship between sexual functioning and sexual satisfaction, the path analysis was used. In order to determine the goodness-of-fit of the model, $\mathrm{X}^{2} / \mathrm{df}$ ratio, Comparative Fit Index (CFI), Goodness-of-Fit Index (GFI) and Adjusted Goodness of Fit Index (AGFI), Root Mean Square Error of Approximation(RMSA) and Non-Normed Fit Index (NFI) are considered were used. According to these indices obtained for the presented model $(\mathrm{NFI}=0.914, \mathrm{RMSA}=0.026$, $\mathrm{AGFI}=0.961, \mathrm{GFI}=0.938, \mathrm{CFI}=0.918, \mathrm{X}^{2} / \mathrm{df}=1.295$ ), it can be said that the model has good fit. The standardized and unstandardized coefficients of determination of the paths are presented in Table 1.

Results showed that the direct effect of sexual functioning on passionate-romantic and open-direct factors of sexual self-schema was positively significant and the direct effect of sexual functioning on embarrassed-conservative factor was negatively related to sexual self-schema. Moreover, the direct effect of sexual functioning on sexual satisfaction was negatively significant. Furthermore, the direct effect of passionate-romantic factor of sexual self-schema on sexual satisfaction was positively significant, while and the direct effect of its embarrassed-conservative factor was negatively significant; However, the open-direct factor of sexual self-schema had no direct effect on sexual satisfaction of students.

Table 1. Summary of path analysis results

\begin{tabular}{ccccc}
\hline Hypotheses (Paths) & $\begin{array}{c}\text { Unstandardized } \\
\text { Coefficient }\end{array}$ & $\begin{array}{c}\text { Standard } \\
\text { Error }\end{array}$ & $\begin{array}{c}\text { Critical Ratio } \\
\text { Coefficient }\end{array}$ & $\begin{array}{c}\text { Standardized } \\
\text { Contion }\end{array}$ \\
\hline Passionate-romantic/Sexual functioning & 0.0970 & 0.019 & $5.084^{*}$ & 0.259 \\
\hline Open-direct/Sexual functioning & 0.066 & 0.019 & $3.541^{*}$ & 0.184 \\
\hline Embarrassed-conservative/Sexual functioning & -0.086 & 0.016 & $-5.461^{*}$ & -0.277 \\
\hline Sexual satisfaction/Sexual functioning & 0.627 & 0.054 & $11.586^{*}$ & 0.516 \\
\hline Sexual satisfaction/Passionate-romantic & 0.588 & 0.137 & $4.293^{*}$ & 0.181 \\
\hline Sexual satisfaction/Open-direct & 0.047 & 0.140 & 0.739 & 0.014 \\
\hline Sexual satisfaction/Embarrassed-conservative & -0.464 & 0.165 & $-2.808^{* *}$ & -0.119 \\
\hline$* * \mathrm{P}<0.01 ; * \mathrm{P}<0.001$ & & & Iranian Journal of
\end{tabular}

**P $<0.01 ; * \mathrm{P}<0.001$ 


\section{Discussion}

This study was conducted to examine the mediating role of sexual self-schema in the relationship of sexual functioning with sexual satisfaction in married female students. according to the results, it can be said that the frequency of sexual activity can also affect sexual satisfaction. With the increase in the length of marriage and possibility reduced sexual attractiveness of the partner, increased age, taking care of children and etc, the frequency of sexual activity decreases which can predict the sexual satisfaction of couples [21].

Many theoretical perspectives believe that the cognitive structures of people evolve through interaction with their environment. These cognitive structures, developed from childhood to adulthood, extend to other areas of communication (such as marital and interpersonal relationships) and interfere with the cognitive structures of others. Selfconcept and positive attitude towards sexual activity can lead to satisfying sexual functioning, while negative attitude along with conflicts and weakness can lead to distress, and sexual problems. Women who describe themselves as passionate/romantic, have a more positive attitude towards sexual issues, and experience arousal, stimulation and orgasm at higher levels and in general, they have better sexual relationships. On the contrary, women embarrassed-conservative women sexually have poor activity and have a more negative attitude towards sexual issues. Women with opendirect schema report sexual desire and behavior like women with passionate/romantic schema, but they have less commitment and less stable relationships [10, 41]. Overall, the findings of this study showed that sexual self-schema is a predictive of sexual functioning and sexual satisfaction, and it plays a mediating role between the two variables.

\section{Ethical Considerations}

\section{Compliance with ethical guidelines}

This study is based on observing all ethical standards, participant satisfaction, confidentiality of data and other important points of research ethics.

\section{Funding}

The study was not sponsored and is part of a first author thesis in the Department of Psychology and Educational Sciences of Tehran South Branch Islamic Azad University.

\section{Authors contributions}

Conceptualization, validation, review and editing: All Authors; Research analysis, resources, data processing, writing, and drafting: Fatemeh Zargari-Nejad; Supervision: Mehrnaz Ahmadi.

\section{Conflicts of interest}

The authors declared no conflicts of interest.

\section{Acknowledgements}

We would like to thank all the participants in the present study and all those involved in the implementation of this research, especially the assistance of the Al-Zahra University officials. 


\section{نقش واسطهاى طرحواره خود جنسى در رابطه بين كنشورى جنسى و رضايت جنسى در دانشجويان زن متأهل}

فاطمه زركرىنزراد' هـ مهرناز احمدى'

ا. كروه روانشناسى، دانشكده علوم تربيتى و روان شناسى، واحد تهران جنوب، دانشُكاه آزاد اسلامى، تهران، ايران.

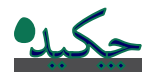

هذافق يُوهش حاضر با هدف بررسي نقش واسطهاي طرحواره خود جنسي در رابطه بين كنشورى جنسي و رضايت جنسي انجام شد.

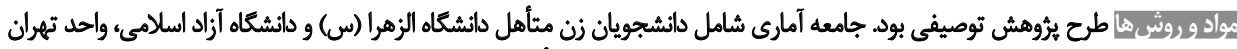

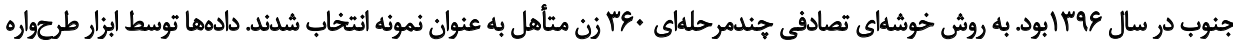

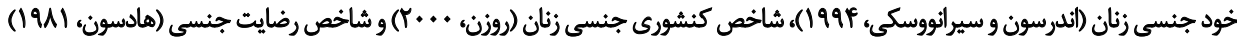
جمعآورى شد.

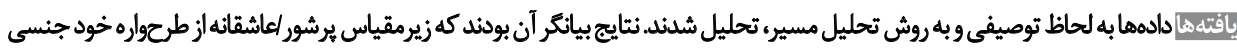

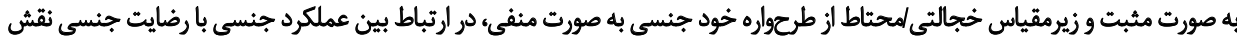

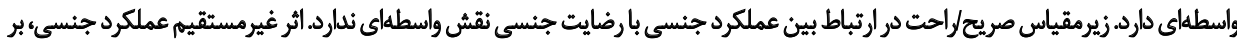

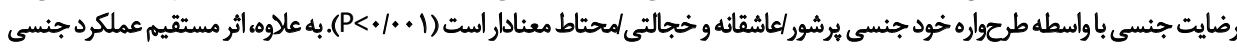

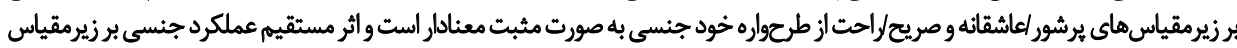

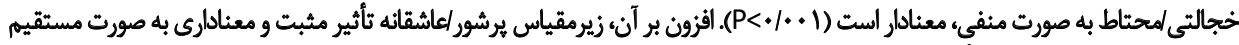

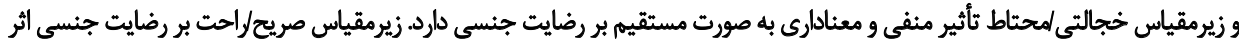

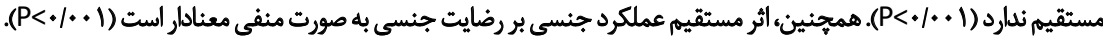

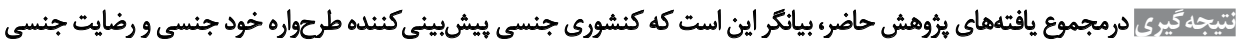

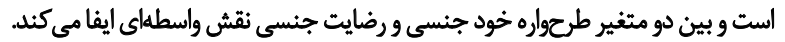

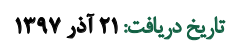

تاريخ بذيرش: 9. شهريور

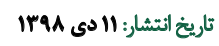

تزديكى و يا آميزش وجود دارد [ب]. يكى از جديدترين نظريه هاي

dalo

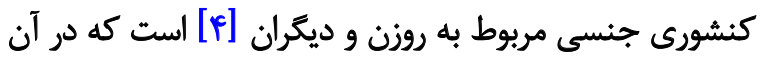

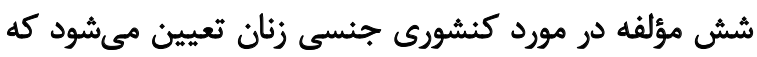

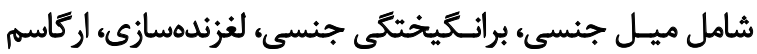

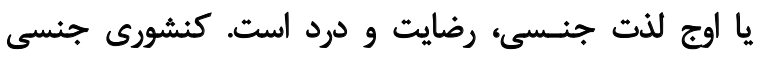

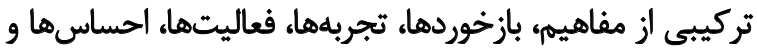

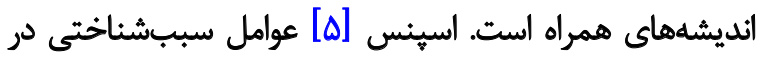

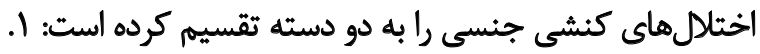

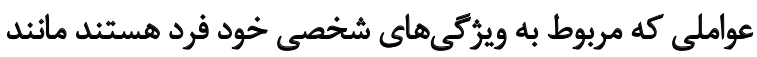

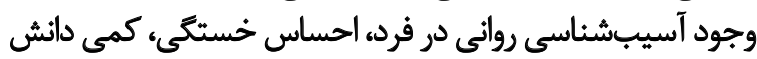

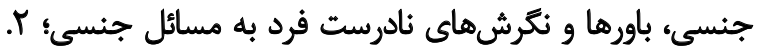

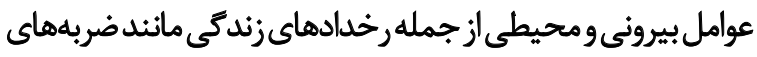

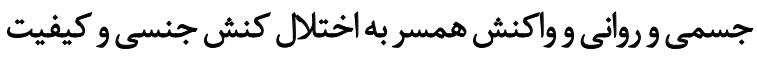

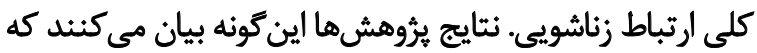

بررسى و مطالعه كنش هاى رابطه جنسى و مؤلفههاى جسمانى

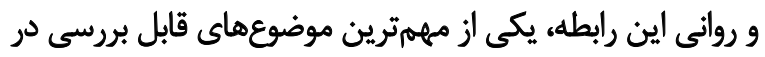

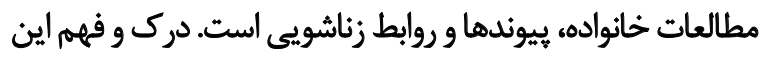

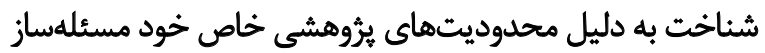

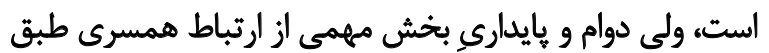

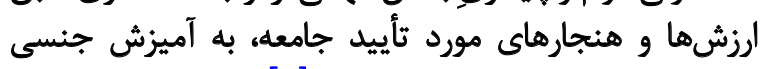

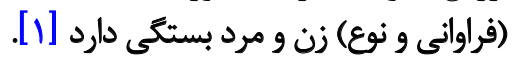

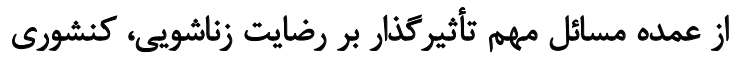

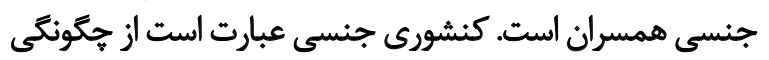

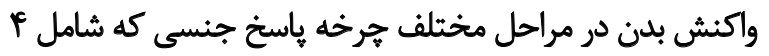

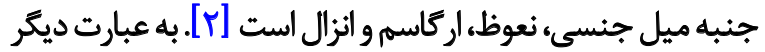

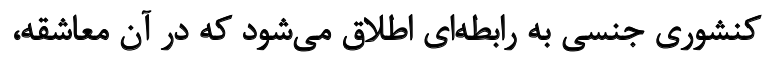




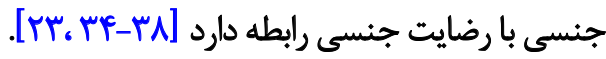
وايشرفتهاى نظرى و تجربى به درك بيشتر شناختهاي مؤثر

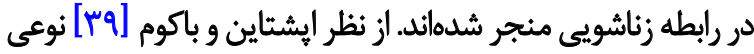

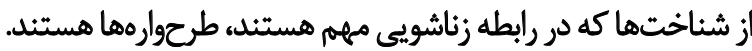

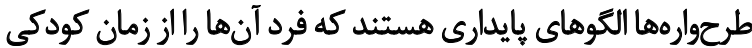

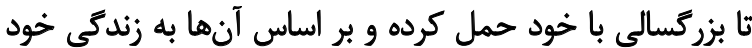

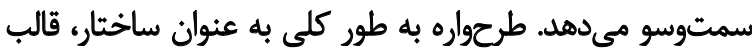

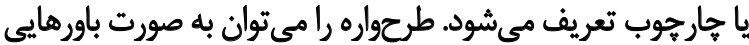

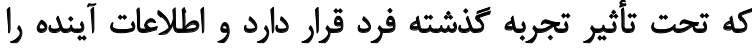

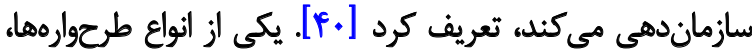

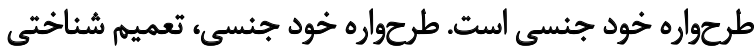

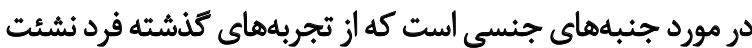

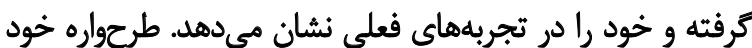

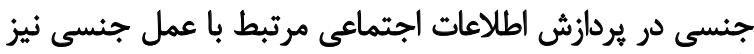

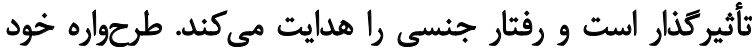

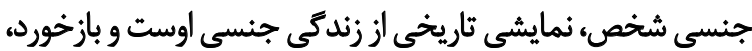

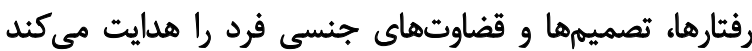

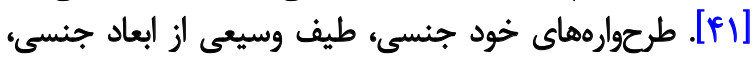

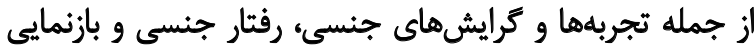

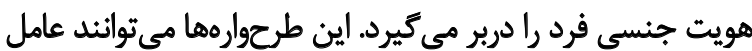

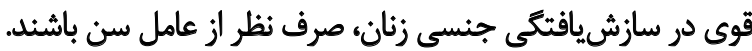

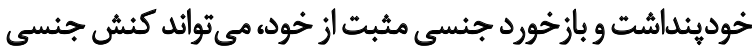

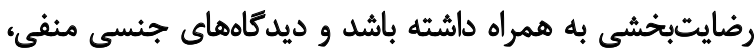

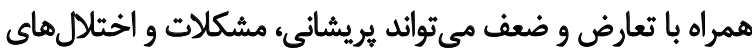

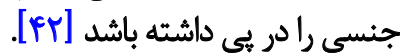
در روابط عاشقانه، طرحوارهها بر نحوه ادراك افراد از اطلاعات.

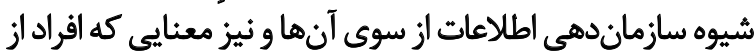

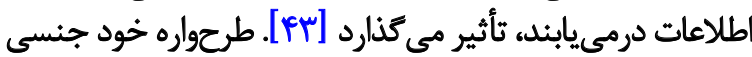

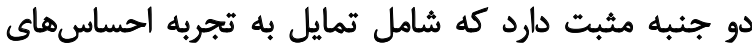

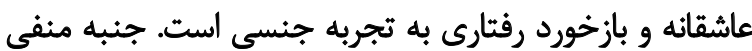

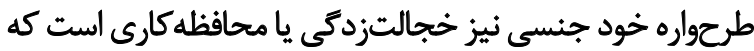

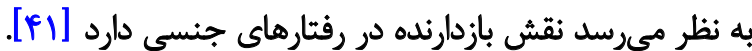

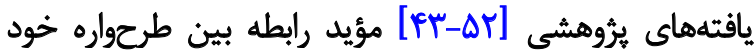

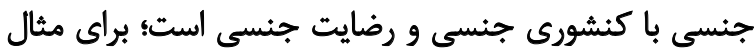

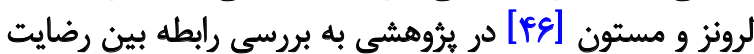

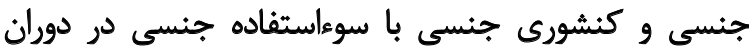

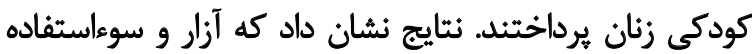

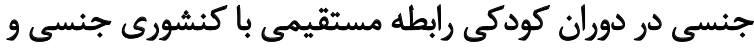

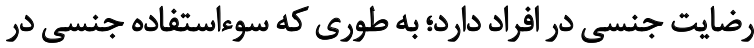

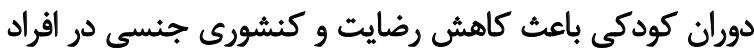

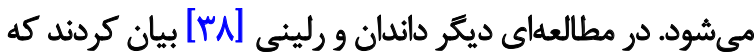

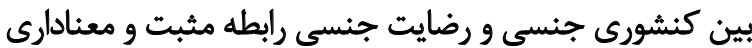

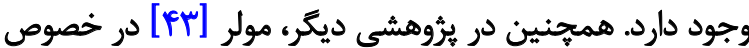

كنشورى جنسى با مؤلفههاى جنسى و غيرجنسى مانند طول

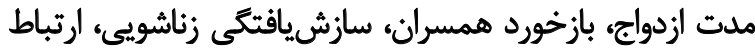

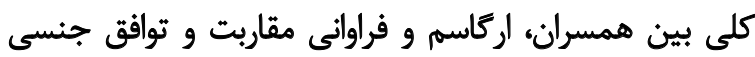

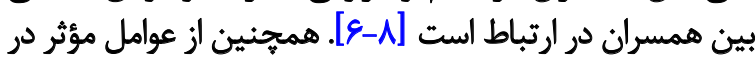

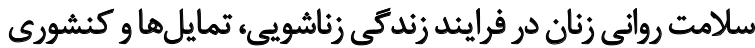

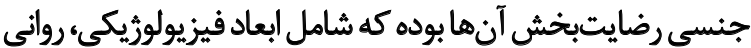

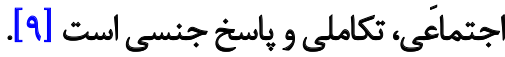

عوامل فيزيولوزيكى، روانشناختى و بينفردى در شكل-

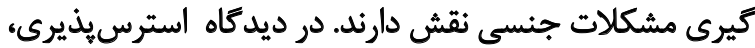

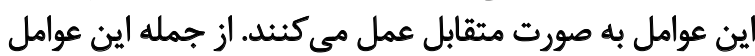

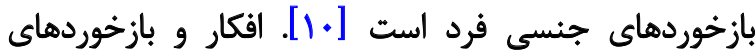

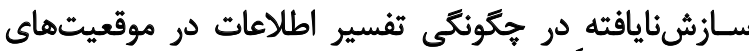

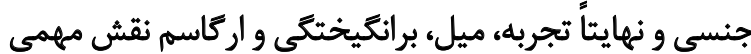

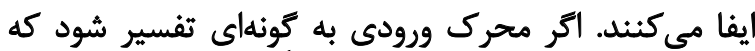

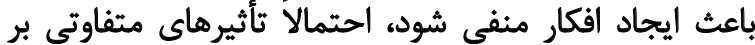

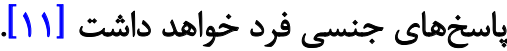

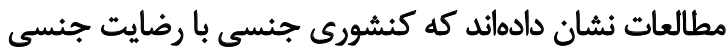

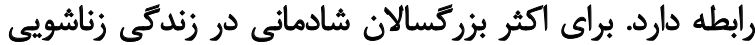

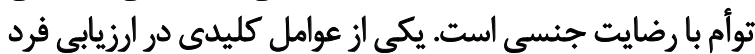

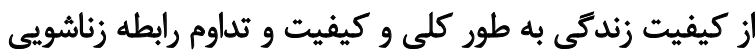

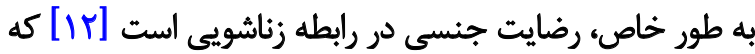

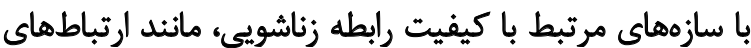

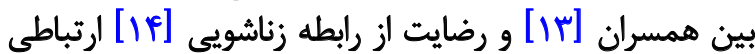

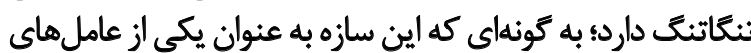

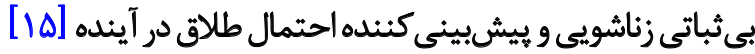
مورد مطالعه برؤهشكران بسيارى قرار كرفته است.

رضايت جنسى مؤلفهاى مرتبط با تمايلهاى جنسى انسان

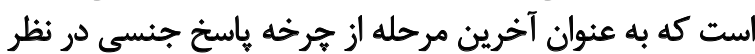

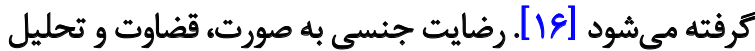

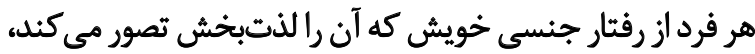

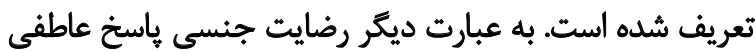

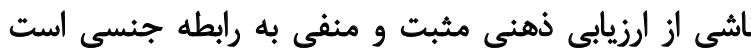

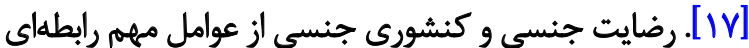

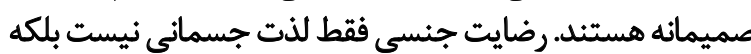

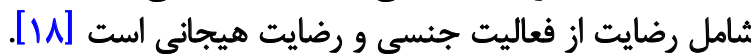

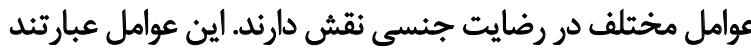

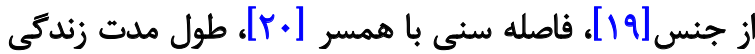

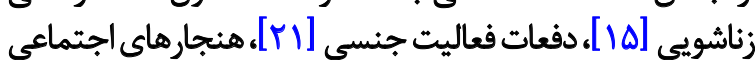

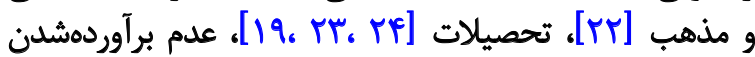

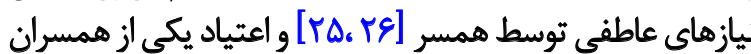

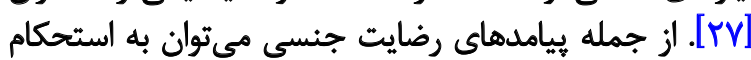

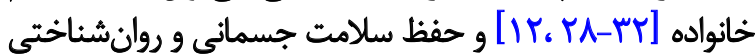
همسران [بس] اشاره كرد. مطالعات نشان دادئ ساداند كه كنشورى 
در هر كلاس زنان مثأهل به صورت داوطلب اقدام به تكميل ابزار

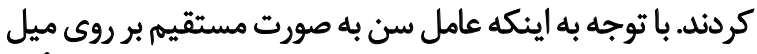

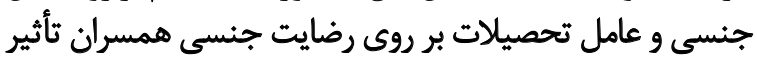

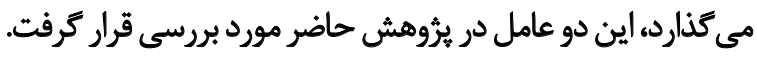
در جدول شماره ا توزيع جمعيتشناختى ارائه شده است

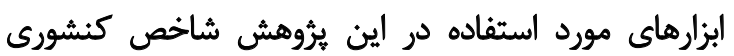

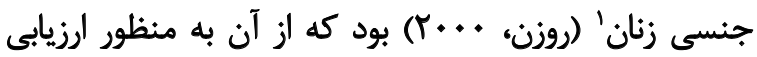

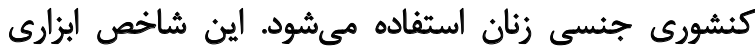

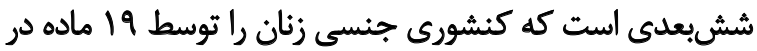

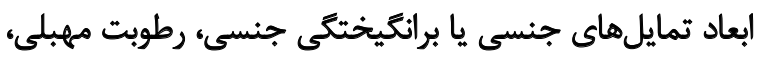

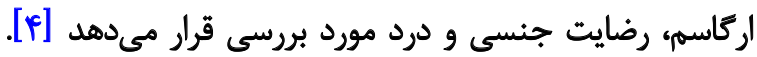

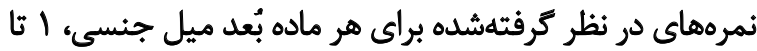

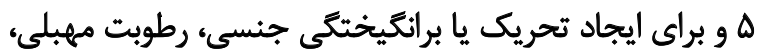

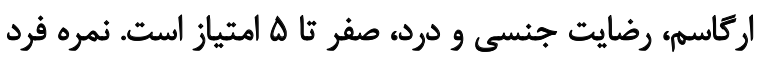

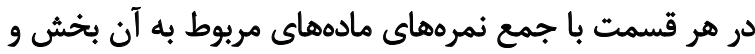

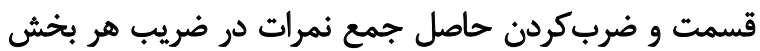

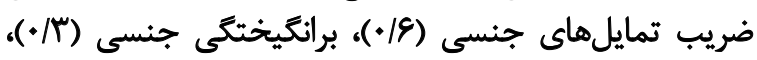

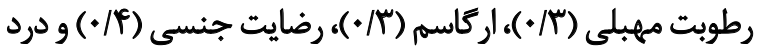

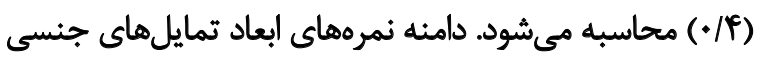

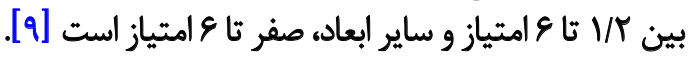

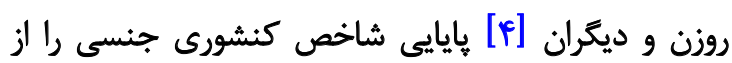

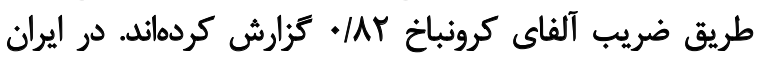

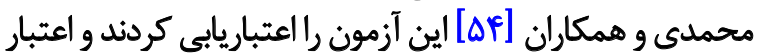

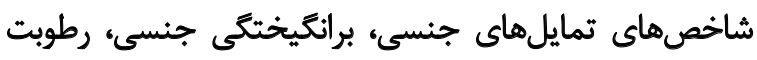

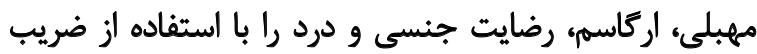

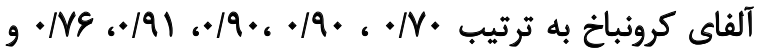

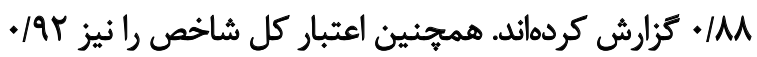

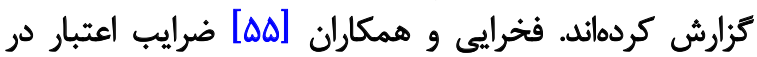

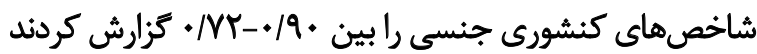

1. Female Sexual Function Index (FSFI)
طرحوارههاى خود جنسى و رضايت جنسى در روابط عاشقانه

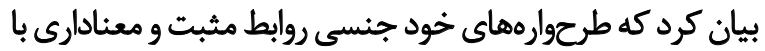

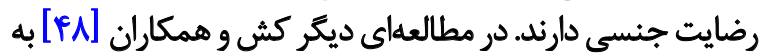

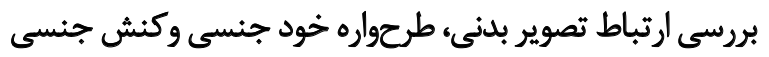

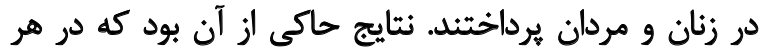

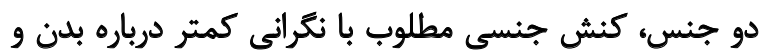

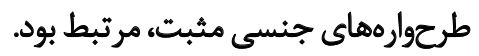

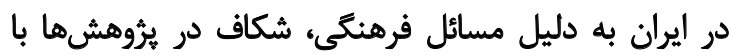

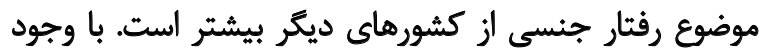

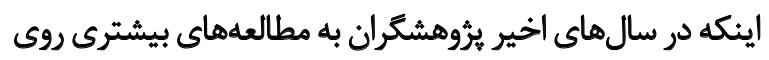

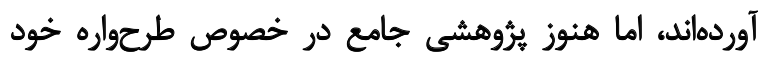

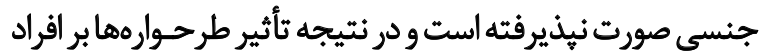

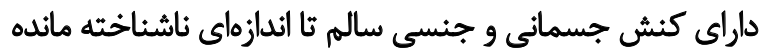

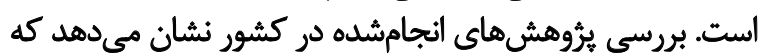

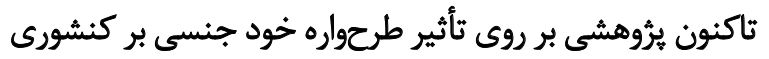

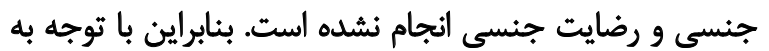

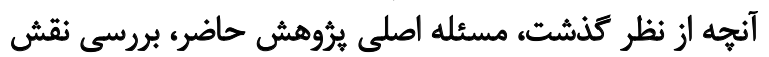

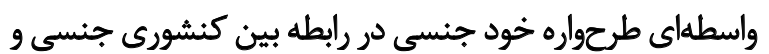
رضايث جنسى بود.

روش ثُروهش حاضر، روش همبستكىى، و و جامعه آمارى

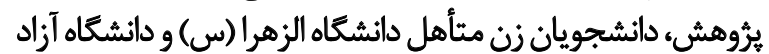

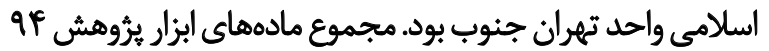

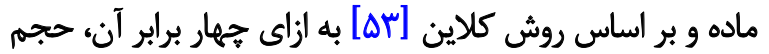

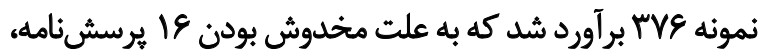

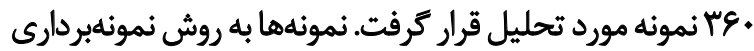

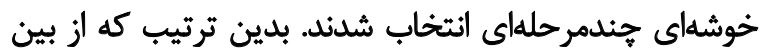

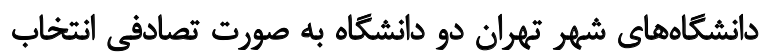

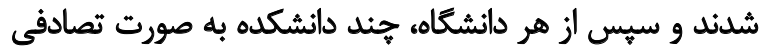

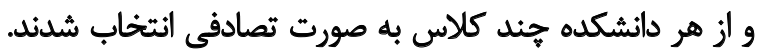

جدول ا. توزيع جمعيتشناختى نمونه

\begin{tabular}{|c|c|c|c|}
\hline \multicolumn{3}{|c|}{ تعداد در مقطع تحصيلى } & \multirow{2}{*}{ سن } \\
\hline دكترى & كارشناسى الرشد & كارشناسي & \\
\hline 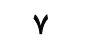 & E & Iro & $M-r$ \\
\hline 11 & $w$ & PA & r \\
\hline$r$ & 18 & 1. & $M F-\Delta C$ \\
\hline \multirow[t]{2}{*}{ M } & 108 & Mr & جمع \\
\hline & re. & & جمع كل \\
\hline
\end{tabular}




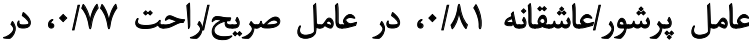

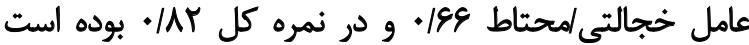

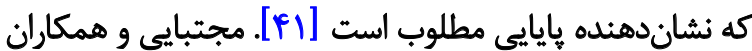

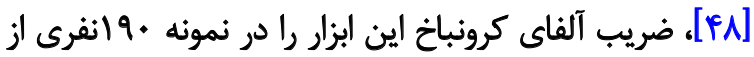

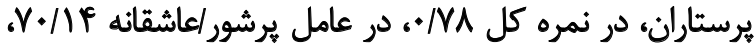

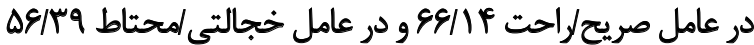

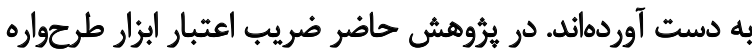

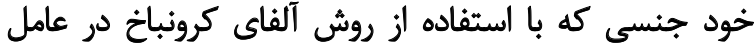

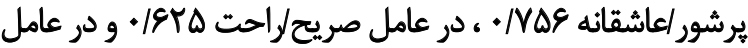

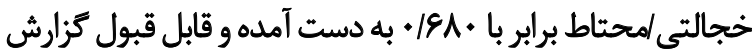

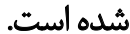

يافتهها

ميانكين توزيع نمرههاى متغيرهاى رضايت جنسى و كنشورى

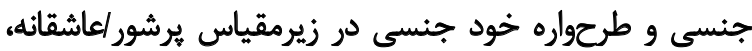

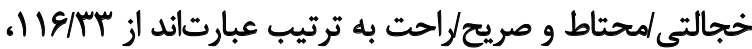
S9/VV

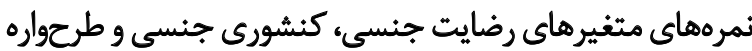

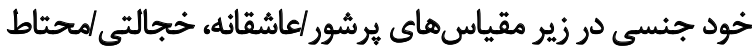

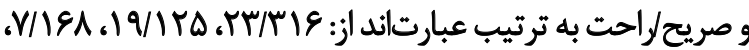

$.9 / 19 \cdot .0 / 97)$

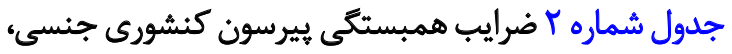

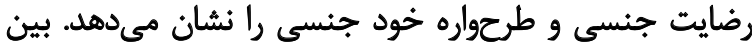

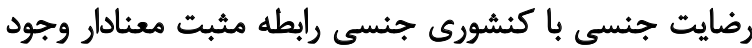

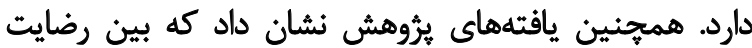

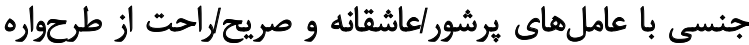

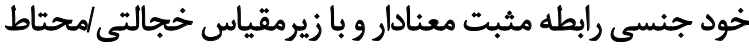
از طرحواره خود جنسى رابطه منفى معناديت معنار وارئ دارد.

براى بررسى نقش واسطهاى طرحواره خود جنسى در رابطه بين كنشورى جنسى و رضايت جنسى از روش برى تحليل مسيل مسير استفاده

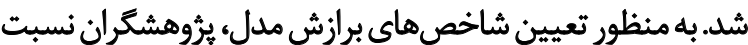

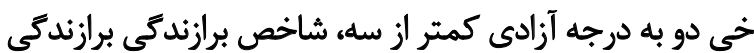

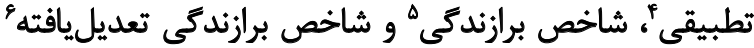

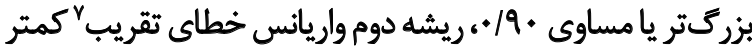

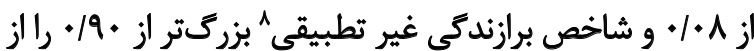

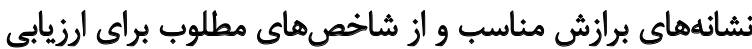

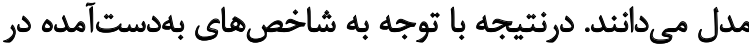

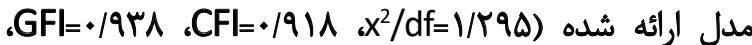

\footnotetext{
4. Comparative Fit Index (CFI)

5. Goodness of Fit Index (GFI)

6. Adjusted Goodness of Fit Index (AGFI)

7. Root Mean Square Error of Approximation (RMSA)

8. Non-Normed Fit Index (NFI)
}

و با استفاده از روش تحليل عاملى شش عامل آن را تأيبيد كردئد.

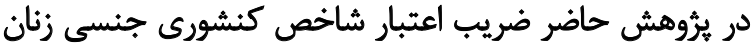

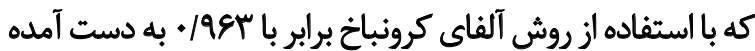

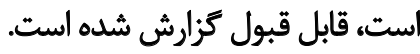

شاخص رضايت جنسيى (هادسون، (141) اين شاخص حاوى ده ماده است كه با مقياس هفتدرجهاى

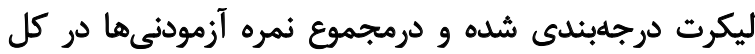

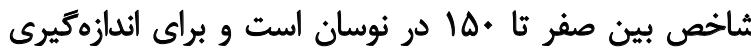

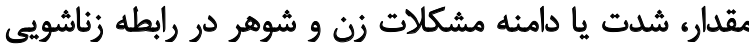

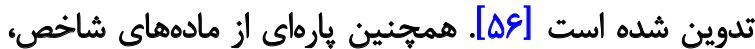

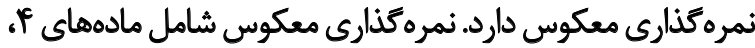

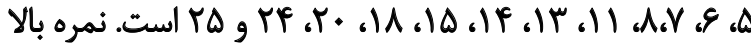

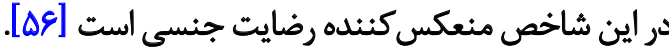

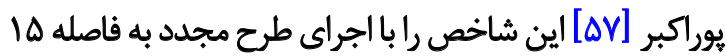

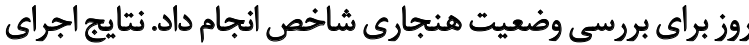

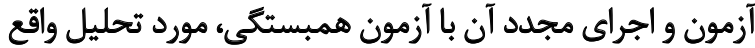

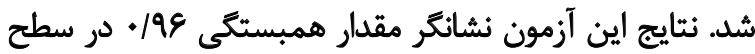

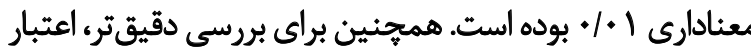

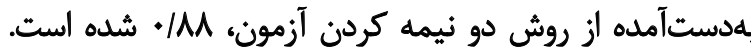

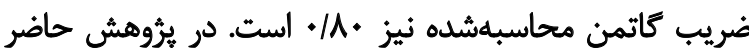

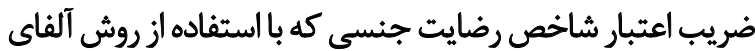
كرونباخ برابر با ArV • به دست آمده است، قابل قبول كزارش شيده اسيت.

\section{ابزار طرحواره خود جنسى" (اندرسون و سيرانوسكى، 199f)} أبزار طرحواره خود جنسى از •ه صفت تشكيل شده است.

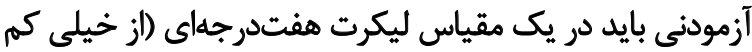

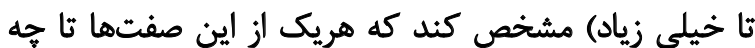

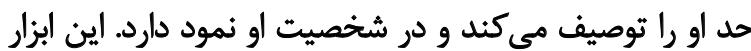

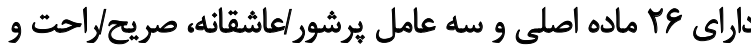

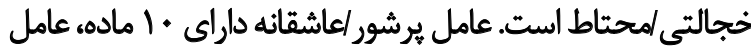

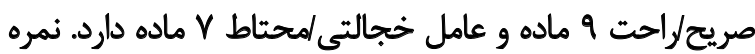

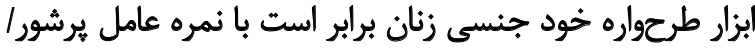

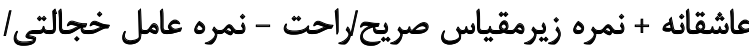

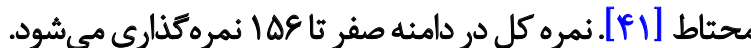

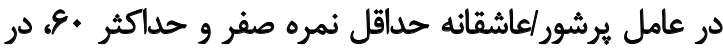

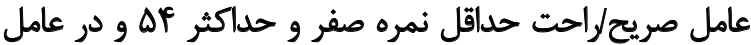

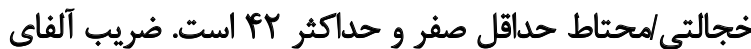

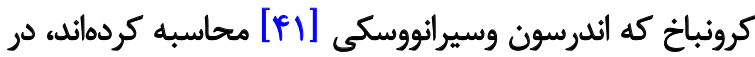

2. Index of Sexual Satisfaction (ISS)

3. The women's Sexual Self-schema Measure 
جدول r. ضرايب همبستَّى كنشورى جنسى، رضايت جنسى و طرحواره جنسى

\begin{tabular}{|c|c|c|c|c|}
\hline$f$ & $r$ & $r$ & 1 & مثغيرها \\
\hline & & & - & كنشورى جنسى \\
\hline & & $=$ & .1098 & رضايت جنسى \\
\hline & - &.$/ M c \cdot$ &.$/ T \Delta 9^{\circ}$ & يرشور/عاشقائه \\
\hline- &.$/ T A A^{*}$ & $\cdot / r \cdot r$ &.$/ M F^{*}$ & صريح/راحت \\
\hline.$-|r \Delta|^{\circ}$ &.$- / 16 * *$ &.$- /$ rav & $-\cdot / r w^{*}$ & خجالتى /محتاط \\
\hline
\end{tabular}

جدول r. خلاصه يافتهاى آزمون تحليل مسير

\begin{tabular}{|c|c|c|c|c|}
\hline تخمين استانداردشده & مقدار بحرانى & خطاى استاندارد & تخمين استانداردينشده & فرضيه ها (مسيرها) \\
\hline . RAQ & $\Delta / \cdot A+*$ & .1 .19 & $1 \% 1.9 \mathrm{~V}$ & عملكردجنسى ـ ـ بيرشور /عاشقائه \\
\hline.$/ M F$ & $r / \Delta H^{\circ}$ & .1 .19 & .1 .98 & 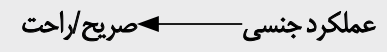 \\
\hline$-\cdot / r w$ & $-\Delta / 48)^{*}$ & .1 .18 & $-* / * N$ & 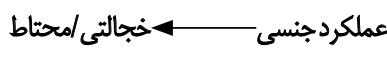 \\
\hline.$/ 018$ & W/ANE* & $.1 . \Delta P$ & . IFT & عملكرد جنسى ـ ــــرضايت جنسى \\
\hline+||$A \mid$ & P/rq" &.$/ 1 \mathrm{rr}$ &.$/ \Delta M$ & 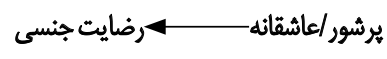 \\
\hline .1 .14 & $\cdot /$ rq $^{2}$ & /If. & 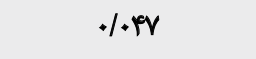 & صريح/لاحت ـرضايت جنسى \\
\hline.$- / 119$ & $-r / A+\Lambda^{*}$ &.$/ 190$ &.$- / 194$ & خجالتى/محتاط| —ــــــرضايت جنسى \\
\hline
\end{tabular}

طرحواره خود جنسى توسط كنشورى جنسى تبيينيذير است.

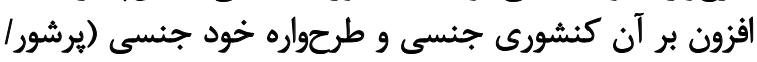

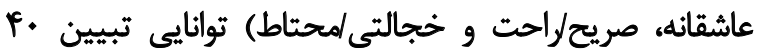

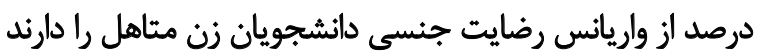

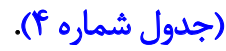

نتايج حاصل از اجراي تحليل مسير براي تعيين نقش واسطهاي

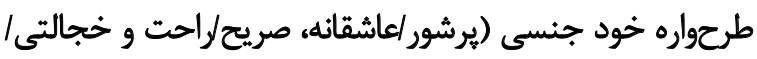

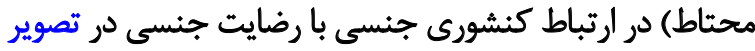

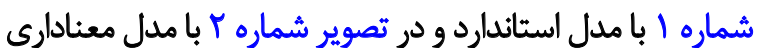
به نمايش درآمده است.

ث

اين يُروهش با هدف دستيابى به يافتههايى كه مي توانند در

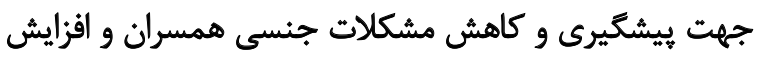

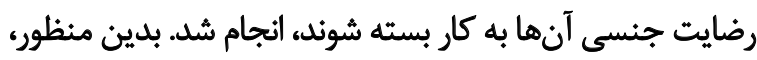

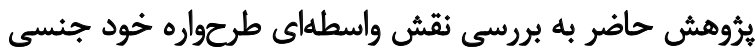

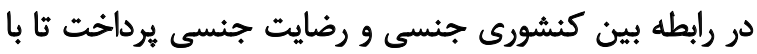
تعيين نقش طرحواره خود جنسى دري در كنشورى جنسى وضى و مقدار

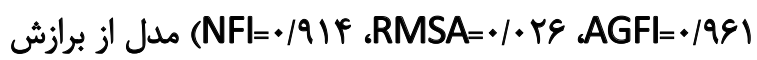

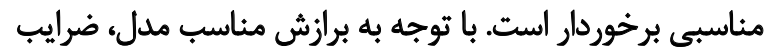

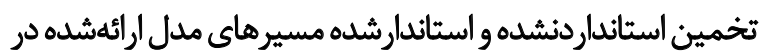
جدول شماره ب منعكس شده است. نتايج بهدستآمده از ضرايب استانداردشده مدل مفهومى تحليل

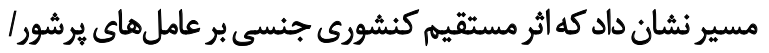

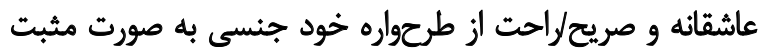

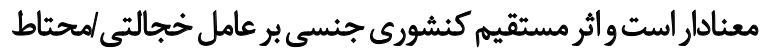

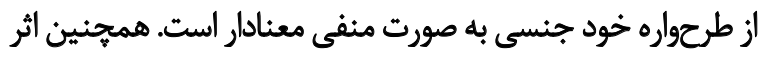

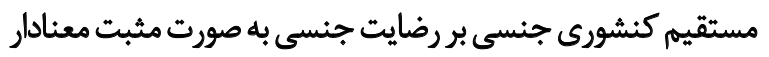

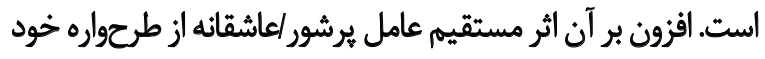

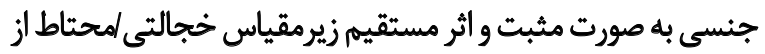

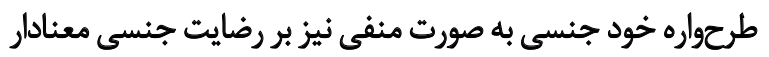

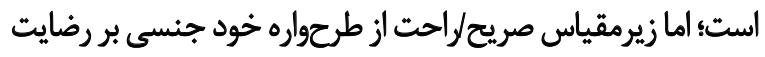
جنسى اثر مستقيم معنادار ندارد. نتايج ضرايب استاندارد كل نشان داد كه هفت درصد از واريانس

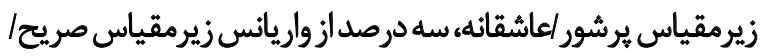

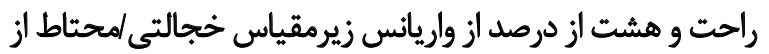


جدول F. ضرايب استاندارد كل، مستقيم و غيرمستقيم در مدل

\begin{tabular}{|c|c|c|c|c|}
\hline واريانس تبيينشده & اثر غير مستقيم & اثر مستقيم & اثر كل & مسيرها \\
\hline \multirow[t]{2}{*}{$+1+9 V$} & & & & بر يرشور /عاثشقانه از \\
\hline & - & . /TAQ &.$/ T \Delta 9$ & عملكرد جنسى \\
\hline \multirow[t]{2}{*}{$.1 \cdot m$} & & & & بر صريع/احت از \\
\hline & - &.$/ M A F$ & . MAr & عملكرد جنسى \\
\hline \multirow[t]{2}{*}{$.1 \cdot W$} & & & & بر خجالئي/محتاط از \\
\hline & - &.$- / r W$ &.$- / r W$ & عملكرد جنسى \\
\hline \multirow[t]{5}{*}{$+R \cdot r$} & & & & بر رضايت جنسي از \\
\hline & $\cdot 1 \cdot \Delta 1^{m *}$ & $. / D \mid E$ & .104 & عملكرد جنسى \\
\hline & - & +||$A \mid$ & $+|M|$ & يرشور/عاشقانه \\
\hline & - & $.1 \cdot$ if & $.1+14$ & صربح/لاحت \\
\hline & - &.$- / 119$ &.$- / 119$ & خجالتى /محتاط \\
\hline
\end{tabular}

اركاسم و يك ينجمم آنها مشكل مربوط به رضايت جنسى دارند.

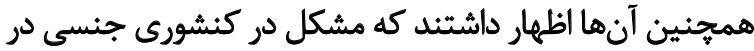

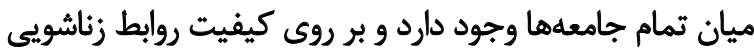

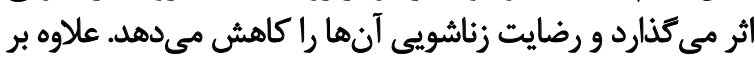

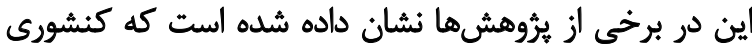

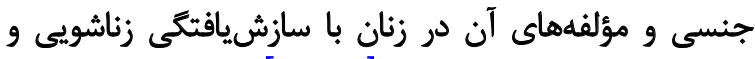

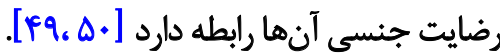

در تبيين اين نتايج مي توان كفت كه رضايت جنسي و عملكرد

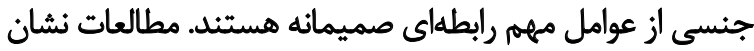

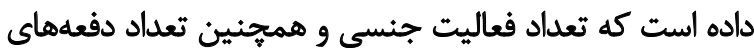

رضايت جنسى و تأثير آن در بهبود روابط زناشويى اهميت آن را برجسته كند.

يافتهها نشان دادند كه بين كنشورى جنسى با رضايت جنسى ، رئى

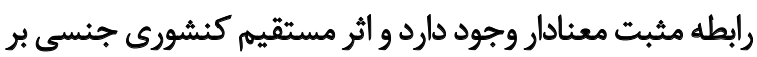

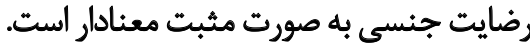

اين يافته با نتايج يثرهشهاي توكل و همكاران، [بr]؛

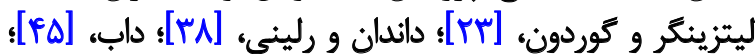

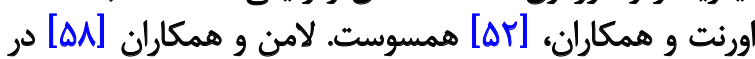

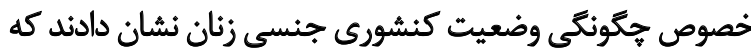

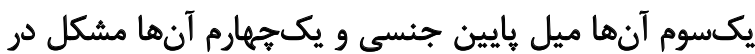

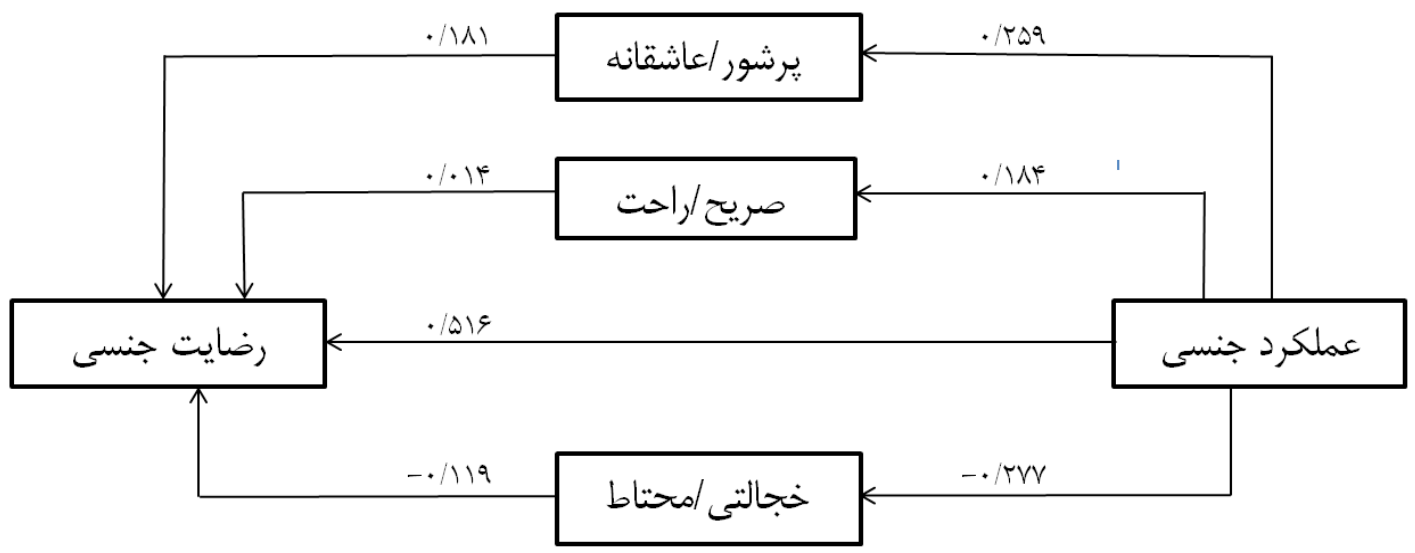




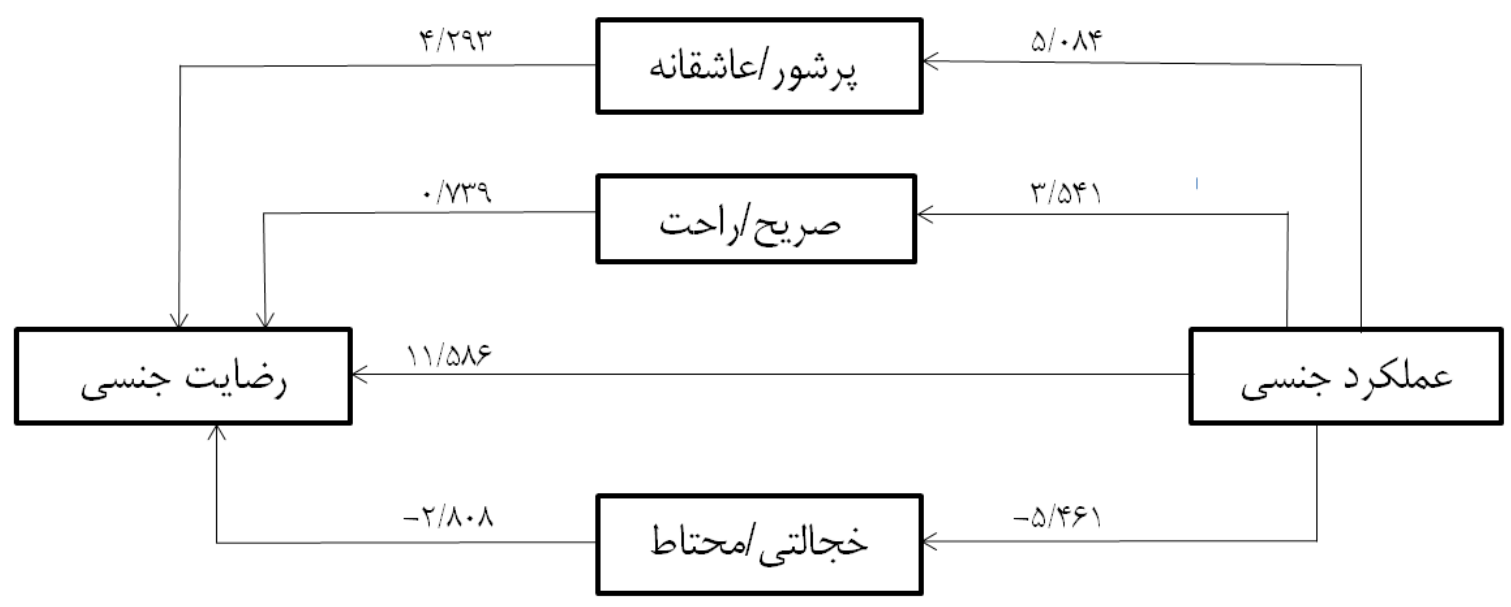

شكست يا موفقيت در روابط زناشويى همسران است. كشاينده

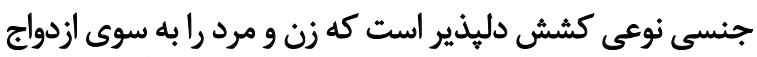

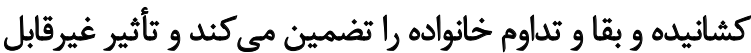
انكارى در زندكى زناشويى و انسجام و بايدارى آن دارد.

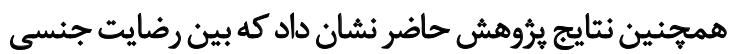

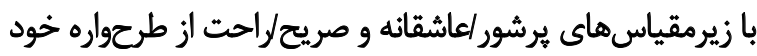

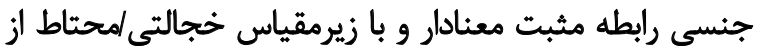

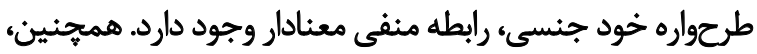

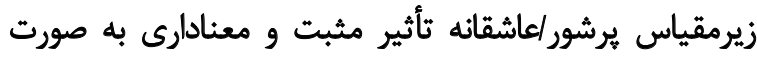

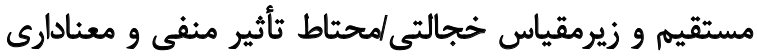

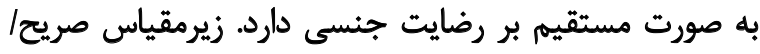

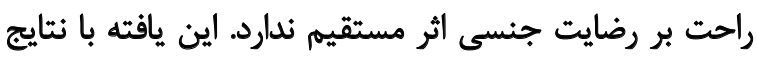

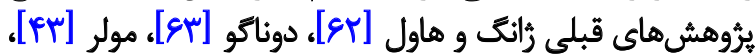

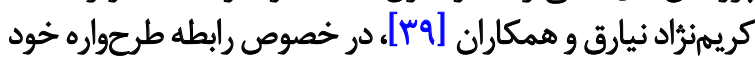

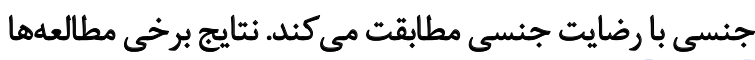

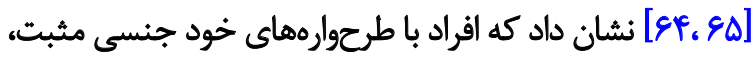

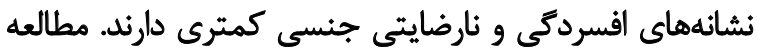

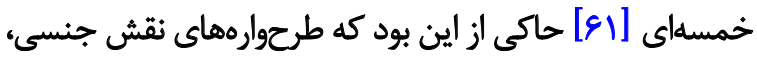

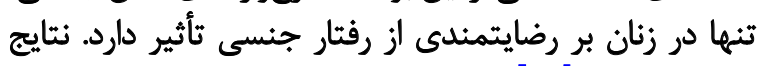

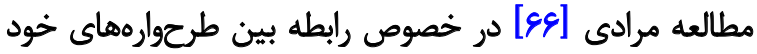

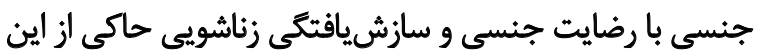

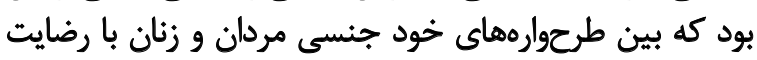

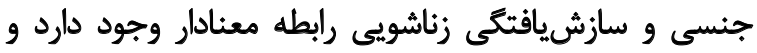

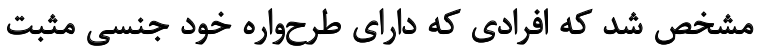

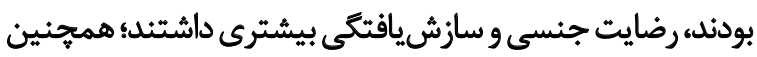

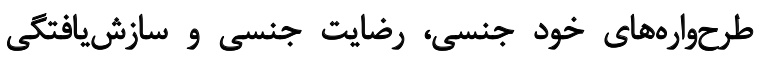
زناشويى را ييشبينى مي كردند.
اركاسم در هر رابطه جنسى نيز با رضايت از روابط جنسى مرتبط

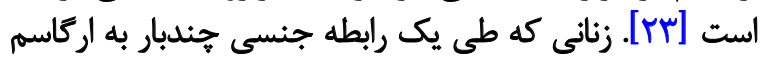

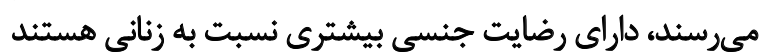

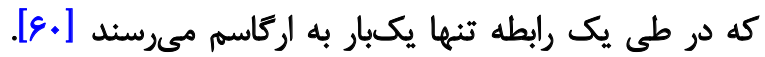

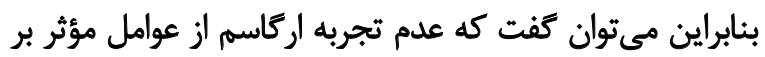

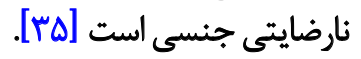

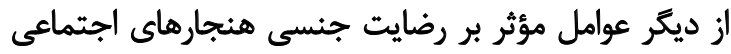

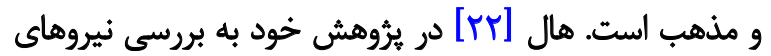

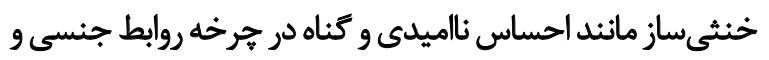

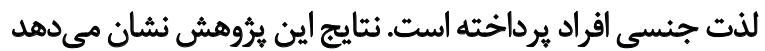

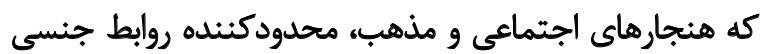

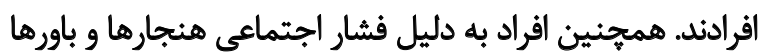

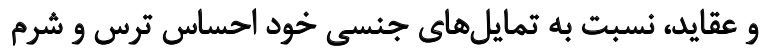

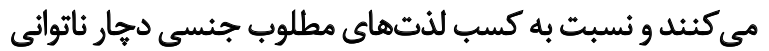

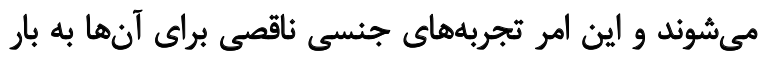

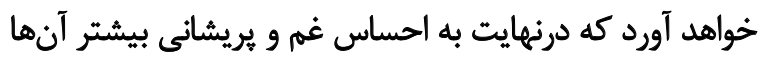

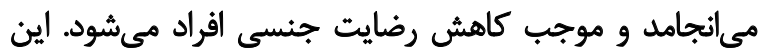

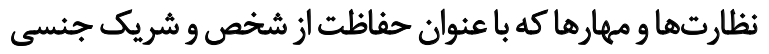

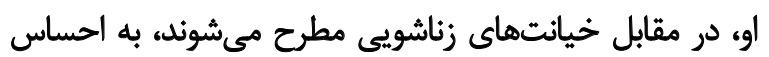

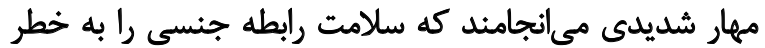
انداخته و مشكلات آن را افزايش مي دهندئ

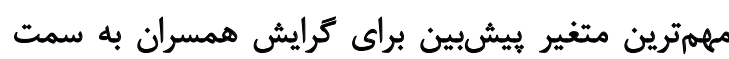

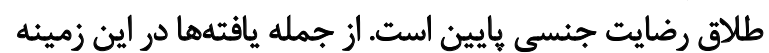

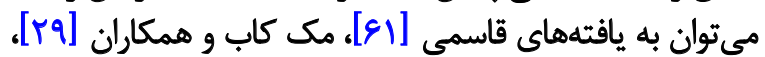

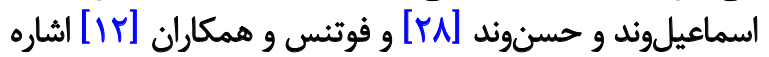

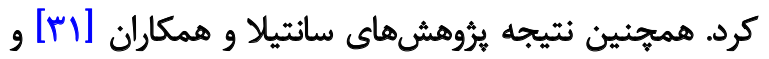

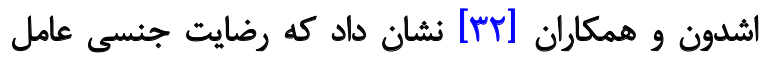


خودينداشت و بازخورد جنسى مثبت از خود مى تواند كنش

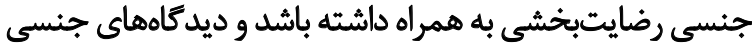

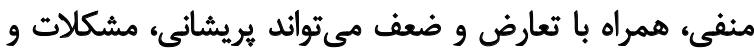

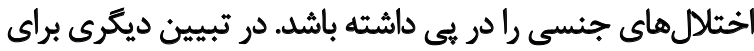

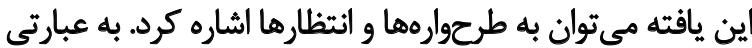

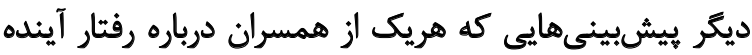

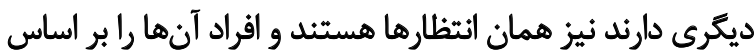

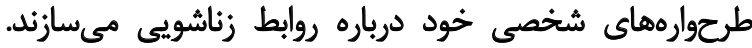

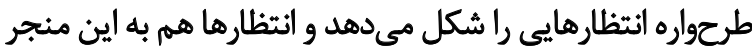

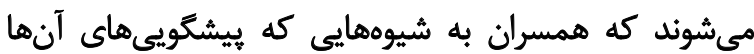

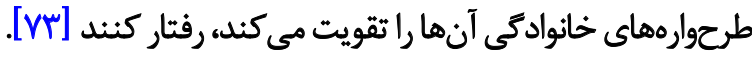
درنهايت نتايج يرؤشش نشان داد كه زيرمقياس يرشور اعاشقانه

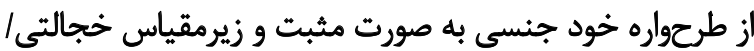

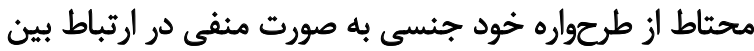

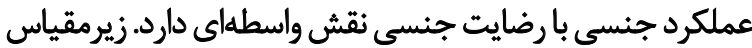

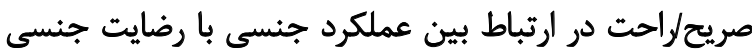

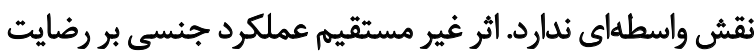

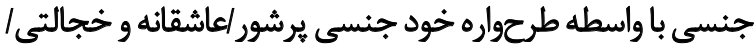

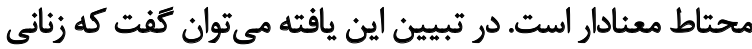

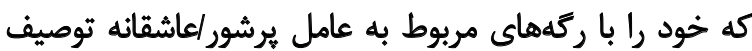

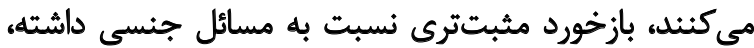

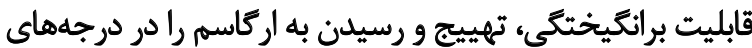

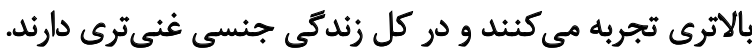

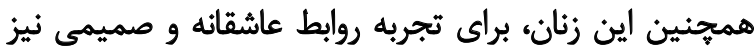

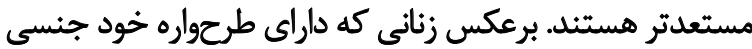

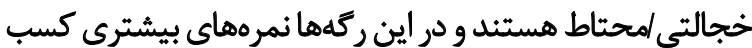

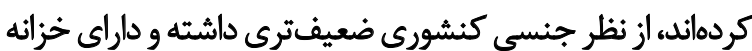

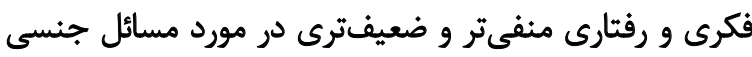
هـيتندي

زنان داراى طرحواره صريح/راحت، تمايل ها و رفتارهاي جنسى كاري

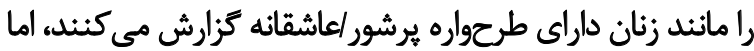

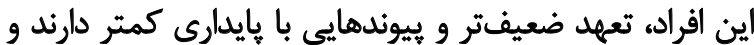

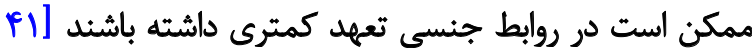

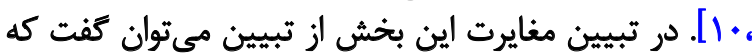

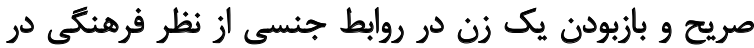

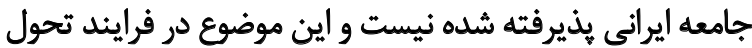

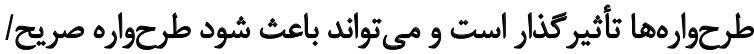

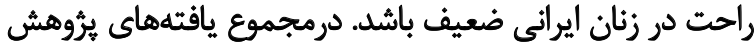

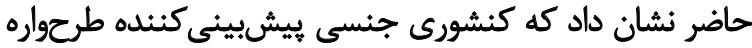

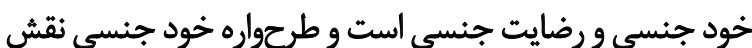
واسطهاي بين دو متغير كنشورى جنسى و وضنى رضايت جنسى ايفا جنسي

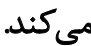

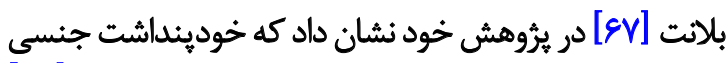

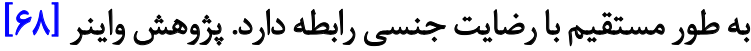

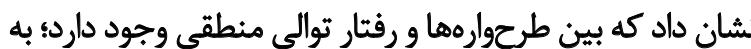

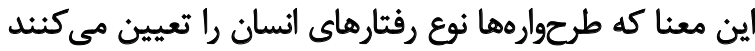

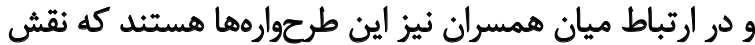

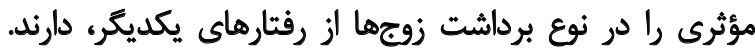

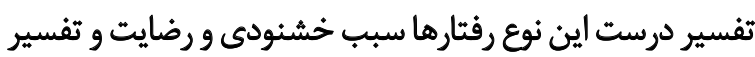
نادرست، سبب عدم رضايت مىشود.

در تبيين اين يافتهها مىتوان كفت كه بسيارى از ديدكاههاى

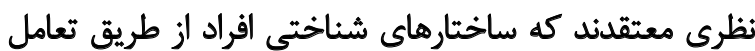

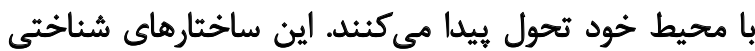

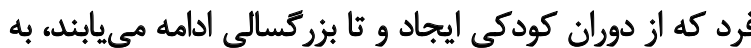

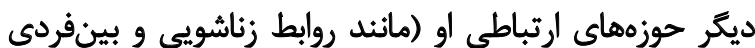

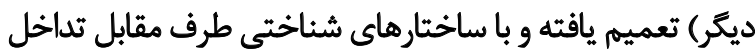

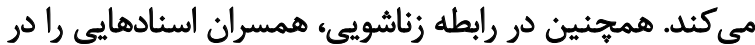

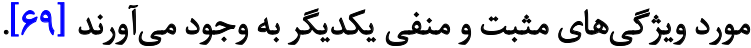

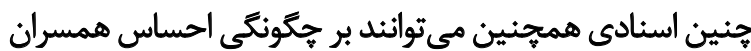

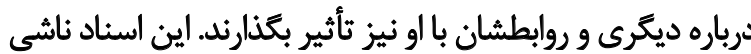

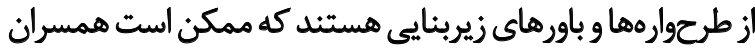

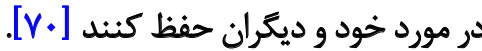

نتايج يُروهش نشان داد كه كنشورى جنسى بازيرمقياس إنهاى

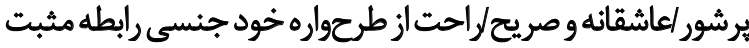

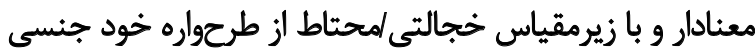

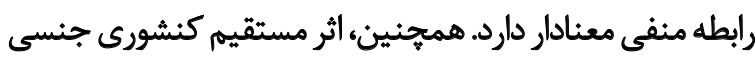

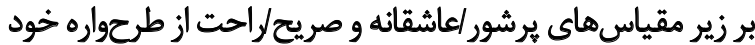
جنسى به صورت مثبت معنادار است و اثر مستقيه كنشئرورى

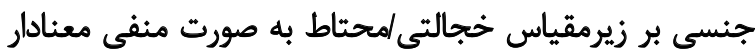

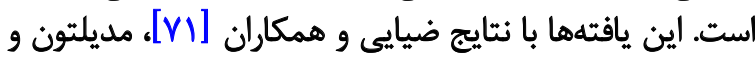

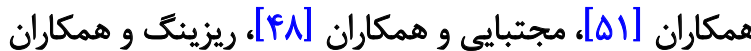

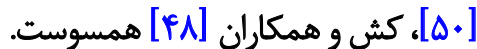
در يروهش رلينى و مستون [VT] ارتباط بين طرحوارههاى

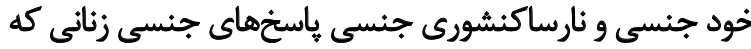

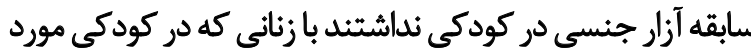

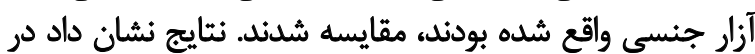

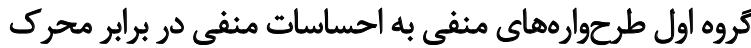

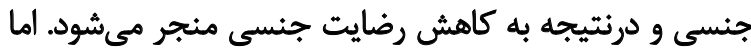

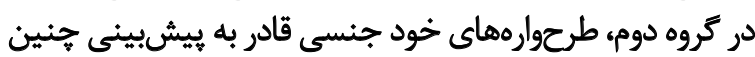

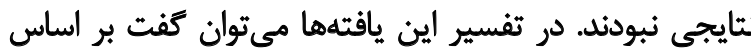

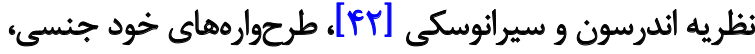

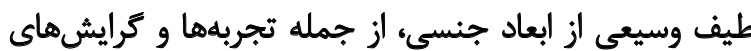

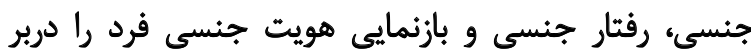

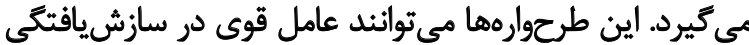
جنسى زنان، صرف نظر از عامل سن باشند. 
مشاوران و بهخصوص مشاوران فعال در حوزه زوجدرمانكرى و

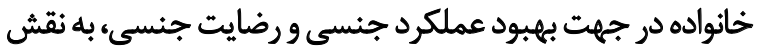

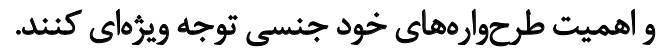

ملاحظاث اخلاقي

\section{ييروى از اصول الخالاق ثوثوهش}

ثرؤوهش حاضر مبتنى بر رعايت كليه موازين اخلاق، رضايت

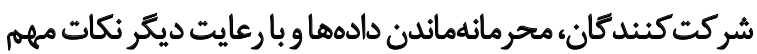

اخلاق يُروهش انجام شده است.

$$
\text { ماهي مالبي }
$$

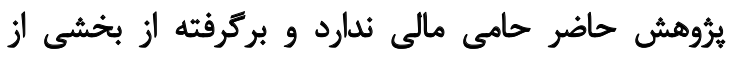

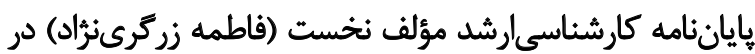

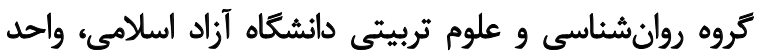

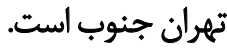

\section{مشاركت نويسند مكان}

مفهومسازى، روششناسى، مرور و ويرايش: همه نويسندكان؛

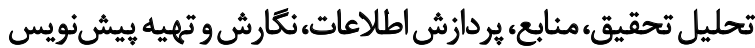

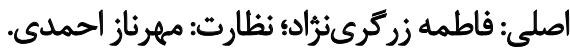

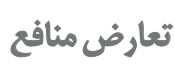

بنا به اظهار نويسندكان، اين مقاله تعارض منافع ندارد.

$$
\text { تشكر و قدروائى }
$$

بدينوسيله از مساعدت مسئولان محترم دانشعاه الزهرا (س)،

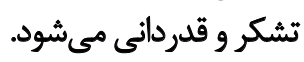

\section{نتيجلتيرى}

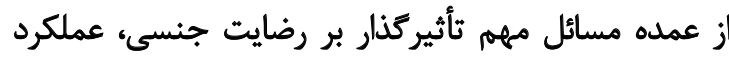

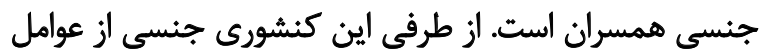

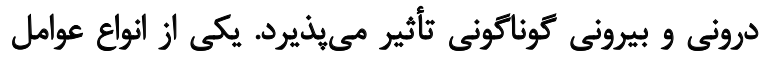

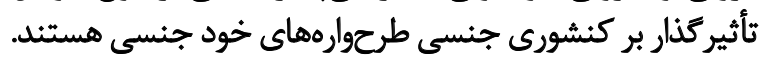

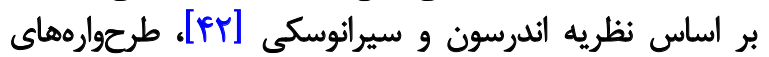

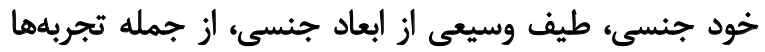

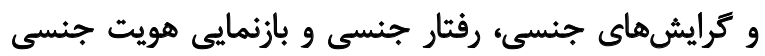

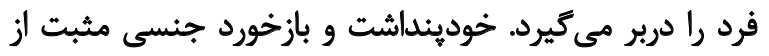

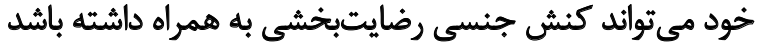

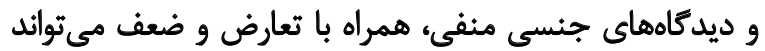

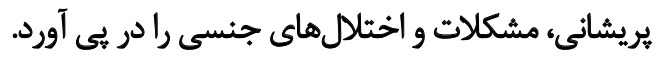

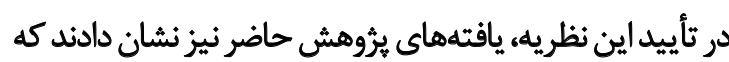

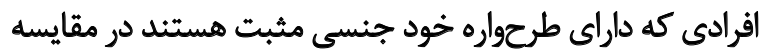

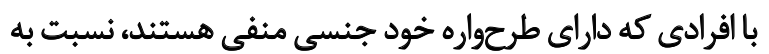

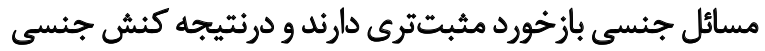

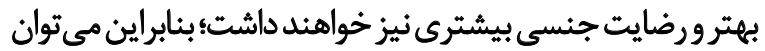

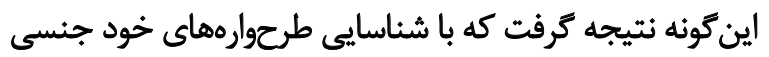

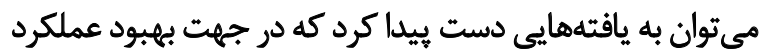

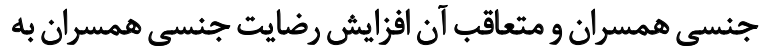

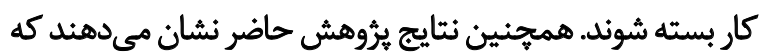

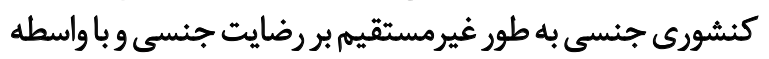

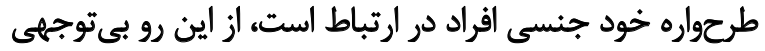

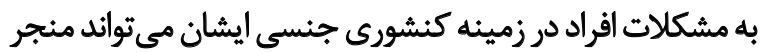

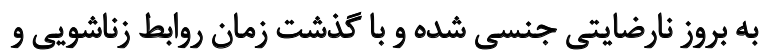
خانوادگى راتحت تأثير قرار دهد.

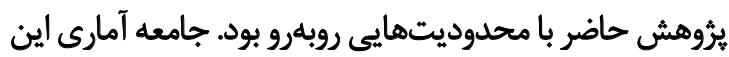

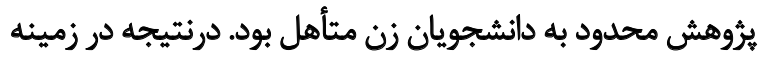

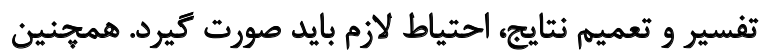

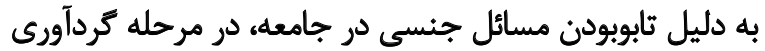

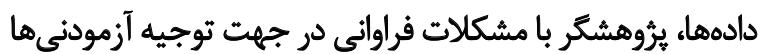

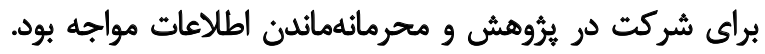

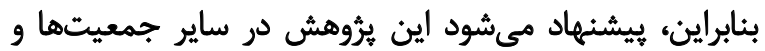

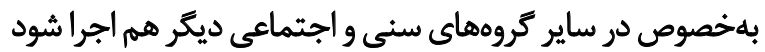

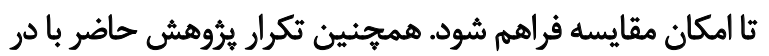

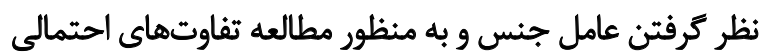

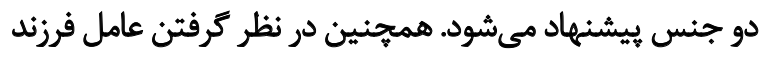
نيز مي تواند به نتايج تكميلى منجر شودي

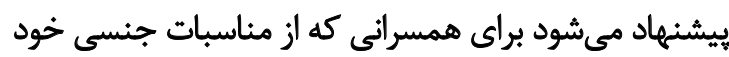

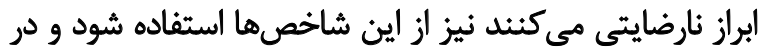

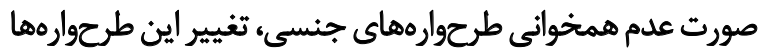

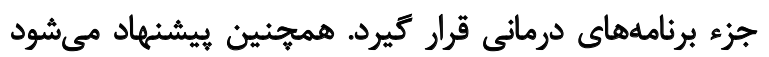




\section{References}

[1] Ismaeli A, Asadollahi A, Bartvand M. [The function of sexual issues in mutual relations of couples in Ahwaz City, Iran (Persian)]. The Journal of Sociology Studies. 2014; 7(22):79-89.

[2] Masters WH, Johnson VE. Human sexual response. $1^{\text {st }}$ edition. United States of America: Ishi Press International; 1966.

[3] Bernhard LA. Sexuality and sexual health care for women. Clinical Obstetrics and Gynecology. 2002; 45(4):1089-98. [DOI:10.1097/00003081-200212000-00017] [PMID]

[4] Rosen R, Brown C, Heiman J, Leiblum S, Meston C, Shabsigh R, et al. The Female Sexual Function Index (FSFI): A multidimensional self-report instrument for the assessment of female sexual function. Journal of Sex \& Marital Therapy. 2000; 26(2):191-208. [DOI:10.1080/009262300278597] [PMID]

[5] Mehrabi F, Dadfar M. [The role of psychological factors in sexual functional disorders (Persian)]. Iranian Journal of Psychiatry and Clinical Psychology. 2003; 9(1):4-11.

[6] Gossman I, Julian D, Mathieu M, Chartrand E. Determinants of sex initation frequencies and sexual satisfaction in long-term couples' relationships. Canadian Journal of Human Sexuality. 2003; 12(3-4):169-81.

[7] Basat Ç. An exploration of marital satisfaction, locus of control, and self-esteem as predictors of sexual satisfaction [MSc. thesis]. Çankaya/Ankara: Middle East Technical University; 2004.

[8] Fisher TD, McNulty JK. Neuroticism and marital satisfaction: The mediating role played by the sexual relationship. Journal of Family Psychology. 2008; 22(1):112-22. [DOI:10.1037/08933200.22.1.112] [PMID]

[9] Jafarpour Ghannhooyeh M, Maroufi M, Molaeinezhad M. [Relationship between sexual self-concept and sexual performance in married women referring to Mobarakeh Health Centers, Iran (Persian)]. Journal of Research Development in Nursing \& Midwifery. 2016; 12(S3):40-7.

[10] Cyranowski JM, Arestad SL, Andersen BL. The role of sexual self-schema in a diathesis-stress model of sexual dysfunction. Applied and Preventive Psychology. 1999; 8(3):217-28. [DOI:10.1016/S0962-1849(05)80078-2]

[11] Spence SH. [Treatment and Sexual Dysfunction (Persian)]. Tehran: Pique Farhang Publishing; 1386

[12] del MarSánchez-Fuentes M, Santos-Iglesias P, Sierra JC. A systematic review of sexual satisfaction. International Journal of Clinical and Health Psychology. 2014; 14(1):67-75. [DOI:10.1016/ S1697-2600(14)70038-9

[13] Nasiri Deh Sorkhi R, Mousavi SF. [The study of some correlative of sexual satisfaction and marital satisfaction in married women of Isfahan City (Persian)]. Rooyesh-e- Ravanshenasi Journal. 2015; 4(2):135-52

[14] Henderson AW, Lehavot K, Simoni JM. Ecological models of sexual satisfaction among lesbin/bisexual and heterosexual women. Archives of Sexual Behavior. 2009; 38(1):50-65. [DOI:10.1007/ s10508-008-9384-3] [PMID] [PMCID]

[15] Edwards JN, Booth A. Sexuality, marriage, and well-being: The middle years. Sexuality Across the Life Course. 1994; 233-59.
[16] World Health Organization. Measuring sexual health: Conceptual and practical considerations and related indicators [Internet] 2010 [Updated 2010]. Available from: https://www.who.int/ reproductivehealth/publications/monitoring/who_rhr_10.12/ en/

[17] Timm TM, Keiley MK. The effects of differentiation of self adult attachment, and sexual communication on sexual and marital satisfaction: A path analysis. Journal of Sex \& Marital Therapy. 2011; 37(3):206-23. [DOI:10.1080/0092623X.2011.564513] [PMID]

[18] Chan JL, Letourneau J, Salem W, Cil AP, Chan SW, Chen LM, et al. Sexual satisfaction and quality of life in survivors of localized cervical and ovarian cancers following fertility-sparing surgery. Gynecologic Oncology. 2015; 139(1):141-7. [DOI:10.1016/j. ygyno.2015.07.105] [PMID]

[19] Guo B, Huang J. Marital and sexual satisfaction in Chinese families: Exploring the moderating effects. Journal of Sex \& Marital Therapy. 2005; 31(1):21-9. [DOI:10.1080/00926230590475224 [PMID]

[20] Heiman JR, Scott Long J, Smith SN, Fisher WA, Sand MS, Rosen RC. Sexual satisfaction and realitionship happiness in midelife and older couples in five countries. Archives of Sexual Behavior 2011; 40:741-53. [DOI:10.1007/s10508-010-9703-3] [PMID]

[21] Klusmann D. Sexual motivation and the duration of partnership. Archives of Sexual Behavior. 2002; 31(3):275-87. [DOI:10.1023/A:1015205020769] [PMID]

[22] Izadi Sh. [Comparison and phenomenology of sex stereotypes in undergraduate students at Tehran's Universities (Persian)] [MSc. thesis]. Marvdasht: Islamic Azad University, Marvdasht Branch; 2015 .

[23] Litzinger S, Gordon KC. Exploring relationships among communication, sexual satisfaction, and marital satisfaction. Journal of Sex \& Marital Therapy. 2005; 31(5):409-24. [DOI:10.1080/00926230591006719] [PMID]

[24] Abdoly M, Pourmousavi L. The relationship between sexual satisfaction and education levels in women. International Journal of Women's Health and Reproduction Sciences. 2013; 1(2):39-44. [DOI:10.15296/ijwhr.2013.07]

[25] Abdi Nia S. [Single girls' strategies for over 29 Years in Tehran for excellence (Persian)] [PhD. dissertation]. Tehran: Allameh Tabataba'i University; 1394

[26] Pourjam S. [Sexual deprivation during marriage in married women in Isfahan (Persian)] [MSc. thesis]. Tehran: Islamic Azad University Central Tehran Branch; 2015.

[27] Rahmani A, Morghati Khoei E, Sadeghi N, Allahgholi L. [Relationship between sexual pleasure and marital satisfaction (Persian)]. Iran Journal of Nursing. 2011; 24(70):82-90.

[28] Samaeilvand N, Hasanvand B. [The relationship between sexual knowledge and marital forgiveness with mental health of married women (Persian)]. Iranian Journal of Health Education and Health Promotion. 2015; 2(4):270-80.

[29] McCabe MP, Sharlip ID, Lewis R, Atalla E, Balon R, Fisher AD, et al. Risk factors for sexual dysfunction among women and men A consensus statement from the fourth international consultation on sexual medicine 2015. The Journal of Sexual Medicine. 2016; 13(2):153-67. [DOI:10.1016/j.jsxm.2015.12.015]

[30] Shakerian A, Nazari AM, Masoomi M, Ebrahimi P, Danai S. Inspecting the relationship between sexual satisfaction and mari- 
tal problems of divorce-asking women in Sanandaj City family courts. Journal of Procedia -Social and Behavioral Sciences. 2014; 114:327-33. [DOI:10.1016/j.sbspro.2013.12.706]

[31] Santtila P, Wager I, Witting K, Harlaar N, Jern P, Johansson A, et al. Discrepancies between sexual desire and sexual activity: Gender differences and associations with relationship satisfaction. Journal of Sex \& Marital Therapy. 2007; 34(1):31-44. [DOI:10.1080/00926230701620548] [PMID]

[32] Ashdown BK, Hackathorn J, Clark EM. In and out of the bedroom: Sexual satisfaction in the marital relationship. Journal of Integrated Social Sciences. 2011; 2(1):40-57.

[33] Paul P. What to expect in sexual therapy. Canada: The University of Toronto; 1998.

[34] Tavakol Z, Mirmolaei ST, Momeni Movahed Z, Mansouri A. [The Relationship between Sexual Function and Sexual Satisfaction in Women Referring to Health Centers in South of Tehran (Persian)]. Avicenna Journal of Nursing and Midwifery Care. 2011; 19(2):50-60.

[35] Dunn KM, Croft P. Satisfaction in the sex life of a general population sample. Journal of Sex \& Marital Therapy. 2000; 26(2):14151. [DOI:10.1080/009262300278542] [PMID]

[36] Carvalheira AA, Leal I. T09-O-04 determinant factors of female sexual satisfaction. Sexologies. 2008; 17(Suppl 1): S117. [DOI:10.1016/S1158-1360(08)72837-8]

[37] Young M, Denny G, Young T, Luquis RR. Sexual satisfaction among married women. American Journal of Health Studies. 2000; 16(2):73-84.

[38] Dundon CM, Rellini AH. More than sexual function: Predictors of sexual satisfaction in a sample of women age 40-70. The Journal of Sexual Medicine. 2010; 7(2):896-904. [DOI:10.1111/j.17436109.2009.01557.x] [PMID]

[39] Karimnejad Niaregh S, Borjeli A, Alavian F, Azartah Shandi F. [The relationship between sexual satisfaction, and sexual schemas with marital satisfaction of female students (Persian)]. Aflak Journal. 2014; 6(20):35-41.

[40] Thimm JC. Mediation of early maladaptive schemas between perceptions of parental rearing style and personality disorder symptoms. Journal of Behavior Therapy and Experimental Psychiatry. 2010; 41(1):52-9. [DOI:10.1016/j.jbtep.2009.10.001] [PMID]

[41] Anderson BL, Cyranowski JM. Women's sexual self-schema. Journal of Personality and Social Psychology. 1994; 67(6):1079100. [DOI:10.1037/0022-3514.67.6.1079]

[42] Cyranowski JM, Anderson BL. Schemas, sexuality, and romantic attachment. Journal of Personality and Social Psychology. 1998; 74(5):1364-79. [DOI:10.1037/0022-3514.74.5.1364]

[43] Mueller K. Sexual self-schemas and sexual satisfaction in romantic relationships [MA. thesis]. Waterloo, Ontario: University of Waterloo; 2013.

[44] Nobre PJ, Pinto-Gouveia J. Dysfunctional sexual beliefs as vulnerability factors for sexual dysfunction. Journal of Sex Research. 2006; 43(1):68-75. [DOI:10.1080/00224490609552300] [PMID]

[45] Dube EW. Relationship satisfaction, sexual satisfaction, sexual behaviors, and perceptions of the father-mother relationship [MSc. thesis]. California: California State University, Long Beach; 2010.
[46] Dortagh Sani S. [Prognosis of sexual satisfaction based on gender role models, body image and personality traits in married students of Bahonar University in Kerman (Persian)] [MSc. thesis]. Shiraz: Shiraz University; 2013.

[47] Claudat K, Warren CS, Durette RT. The relationships between body surveillance, body shame, and contextual body concern during sexual activities in ethnically diverse female college students. Body Image. 2012; 9(4): 448-454. [DOI:10.1016/j.bodyim.2012.05.007] [PMID]

[48] Mojtabaee M, Saberi H, Alizadeh A. [Women sexual function: The role of sexual - self schema and body image (Persian)]. Quarterly Journal of Health Psychology. 2014; 3(9):52-64.

[49] Rezaei Nasab F, Abbasi I, Naziry Gh, Barzegar M. [The determination of early maladaptive schemas in women with addictive relationships (Persian)]. Sociology of Women (Journal of Woman and Society). 2013; 4(2):83-108.

[50] Reissing ED, Laliberté GM, Davis HJ. Young women's sexual adjustment: The role of sexual self-schema, sexual self-efficacy, sexual aversion and body attitudes. The Canadian Journal of Human Sexuality. 2005; 14(3-4):77-85.

[51] Middleton LS, Kuffel SW, Heiman JR. Effects of experimentally adopted sexual schemas on vaginal response and subjective sexual arousal: A comparison between women with sexual arousal disorder and sexually healthy women. Archives of Sexual Behavior. 2008; 37(6):950-61. [DOI:10.1007/s10508-007-9310-0] [PMID]

[52] Ornat L, Martínez-Dearth R, Muñoz A, Franco P, Alonso B, Tajada $\mathrm{M}$, et al. Sexual function, satisfaction with life and menopausal symptoms in middle-aged women. Maturitas. 2013; 75(3):2619. [DOI:10.1016/j.maturitas.2013.04.007] [PMID]

[53] Kline RB. Principles and practice of structural equation modeling. New York: Guilford Publications; 2011.

[54] Mohammadi Kh, Heydari M, Faghihzadeh S. [The Female Sexual Function Index (FSFI): Validation of the Iranian version (Persian)]. Payesh. 2008; 7(3):269-78. http://payeshjournal.ir/ article-1-658-fa.html

[55] Fakhri A, Mohammadi Zeidi I, Pakpour Haji Agha A, Morshedi H, Mohammad Jafari R, Ghalambor Dezfooli F. [Psychometric properties of Iranian version of female sexual function index (Persian)]. Jundishapur Scientific Medical Journal. 2011; 10(4):345-54.

[56] Hudson WW, Harrison DF, Crosscup PC. A short-form scale to measure sexual discord in dyadic relationships. Journal of Sex Research. 1981; 17(2):157-74. [DOI:10.1080/00224498109551110]

[57] Pouurakbar S. [Surveying the role of personality traits in exhalation of the relationship between sexual satisfactions with marital satisfaction (Persian)] [PhD. dissertation]. Tehran: University of Tarbiat Moddares; 2011.

[58] Laumann EO, Nicolosi A, Glasser DB, Paik A, Gingell C, Moreira E, et al. Sexual problems among women and men aged $40-80 \mathrm{y}$ : Prevalence and correlates identified in the global study of sexual attitudes and behaviors. International Journal of Impotence Research. 2005; 17:39-57. [DOI:10.1038/sj.ijir.3901250] [PMID]

[59] Poyner-Del Vento PW, Cobb RJ. Chronic stress as a moderator of the association between depressive symptoms and marital satisfaction. Journal of Social and Clinical Psychology. 2011; 30(9):905-36. [DOI:10.1521/jscp.2011.30.9.905]

[60] Greenberg JS, Bruess CE, Heffner D. Exploring the dimension of human sexuality. Burlington: Jones and Bartlett; 2002. 
[61] Khamsehei A. [Study on the sexual behavior and gender role stereotypes of the married college students in Iran: Comparing sexual behavior of female and male students in the family (Persian)]. Journal of Family Research. 2007; 2(8):327-39.

[62] Zhang JW, Howell RT. Do time perspectives predict unique variance in life satisfaction beyond personality traits? Personality and Individual Differences. 2011; 50(8):1261-6. [DOI:10.1016/j. paid.2011.02.021]

[63] Donaghue N. Body satisfaction, sexual self-schemas and subjective well-being in women. Body Image. 2009; 6(1):37-42. [DOI:10.1016/j.bodyim.2008.08.002] [PMID]

[64] Mueller K, Rehman US, Fallis EE, Goodnight JA. An interpersonal investigation of sexual self-schemas. Archives of Sexual Behavior. 2016; 45(2):281-90. [DOI:10.1007/s10508-015-0638-6] [PMID]

[65] Carpenter KM, Andersen BL, Fowler JM, Maxwell GL. Sexual self schema as a moderator of sexual and psychological outcomes for gynecologic cancer survivors. Archives of Sexual Behavior. 2009; 38(5):828-41. [DOI:10.1007/s10508-008-9349-6] [PMID] [PMCID]

[66] Moradi Organi E. [The relationship between sexual schemas with sexual satisfaction and marital adjustment in married cultural Falavarjan (Persian)] [MSc. thesis]. Isfahan: University of Isfahan; 2012.

[67] Blunt H. "People aren't mind readers": A study of sexual selfconcept, partner communication, and sexual satisfaction $[\mathrm{PhD}$ dissertation]. Tampa, FL: University of South Florida; 2012.

[68] Weiner B. Human motivation. New York, NY: Rinehart \& Winston; 1980 .

[69] Epstein N, Baucom DH. Cognitive factors in marital disturbance. In: Dobson KS, Kendall PC, editors. Personality, Psychopathology, and Psychotherapy Series, Psychopathology and Cognition. Cambridge: Academic Press; 1993.

[70] Dattilio FM, Epstein NB. Cognitive-behavioral couple and family therapy. New York: The Guilford Press; 2010.

[71] Ziyayi P, Sepehri Shamlou Z, Mashhadi A. [The relationship between activating cognitive schemas, negative automatic thoughts and sexual arousal in married female college students (Persian)]. Journal of Clinical Psychology Andishe va Raftar. 2013; 8(28):17-26.

[72] Rellini AH, Meston CM. Sexual self-schemas, sexual dysfunction, and the sexual responses of women with a history of childhood sexual abuse. Archives of Sexual Behavior. 2011; 40(2):35162. [DOI:10.1007/s10508-010-9694-0] [PMID] [PMCID]

[73] Whisman MA, Delinsky SS. Marital satisfaction and an information-processing measure of partner-schemas. Cognitive Therapy and Research. 2002; 26(5):617-27. [DOI:10.1023/ A:1020305226067] 
This Page Intentionally Left Blank 